J Org Chem. 2018 April 06; 83(7): 4131-4139. doi:10.1021/acs.joc.8b00027.

\title{
Synthesis of $1,1^{\prime}$-Diarylethanes and Related Systems by Displacement of Trichloroacetimidates with Trimethylaluminum
}

\author{
Nivedita S. Mahajani and John D. Chisholm ${ }^{*}$ \\ Department of Chemistry, Syracuse University, 1-014 Center for Science and Technology, \\ Syracuse, NY 13244
}

\begin{abstract}
Benzylic trichloroacetimidates are readily displaced by trimethylaluminum under Lewis acid promoted conditions to provide the corresponding methyl substitution product. This method is a convenient way to access $1,1^{\prime}$-diarylethanes and related systems, which play a significant role in medicinal chemistry, with a number of systems owing their biological activity to this functionality. Most benzylic substrates undergo ready displacement, with electron deficient systems being the exception. Use of an enantiopure imidate showed significant racemization, implicating the formation of a cationic intermediate.
\end{abstract}

\section{Graphical Abstract}

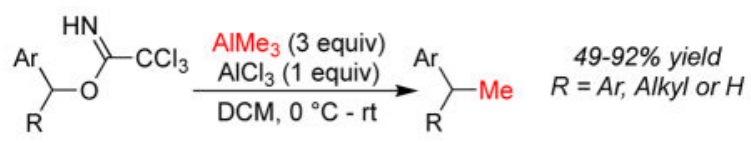

Trichloroacetimidates are often used to activate alcohols as leaving groups, commonly being displaced by heteroatom nucleophiles like alcohols, ${ }^{1}$ carboxylic acids, ${ }^{2}$ thiols ${ }^{3}$ and amines. ${ }^{4}$ This chemistry is especially important in the synthesis of carbohydrates. ${ }^{5}$ Imidate displacement is facilitated by rearrangement of the leaving group to trichloroacetamide, providing an additional thermodynamic driving force to drive the displacement. The imidate structure also features a basic nitrogen, which is readily activated by catalytic amounts of Brønsted or Lewis acids. Trichloroacetimidates are easily formed from alcohols from the inexpensive trichloroacetonitrile, and the imidates can be generated in situ, ${ }^{6}$ providing a method to use inexpensive and readily available alcohols as alkylating agents under mild conditions.

While the use of imidates to form ethers, sulfides and substituted amines has become well known, it is more rare to displace trichloroacetimidates with carbon nucleophiles. As trichloroacetimidates are typically activated with Lewis acids, a carbon nucleophile must be

\footnotetext{
Corresponding Author: jdchisho@syr.edu.

Notes

The authors declare no competing financial interest.

Supporting Information Available

Copies of ${ }^{1} \mathrm{H}$ and ${ }^{13} \mathrm{C}\left\{{ }^{1} \mathrm{H}\right\}$ NMR spectra and chiral HPLC data for compound 16. This material is available free of charge via the Internet at http://pubs.acs.org.
} 
compatible with these conditions. Typically, these substitutions are limited to Friedel-Crafts type processes with electron rich aromatic rings acting as nucleophiles, ${ }^{3 \mathrm{~h}, 7}$ although there have been reports of silyl enol ethers, allylsilanes and allylstannanes being effective nucleophiles under Lewis acid catalysis. ${ }^{6 b, 8}$ Currently there are no reports on the displacement of trichloroacetimidates with stoichiometric amounts of alkylmetal reagents.

Recently investigations into increasing the scope of nucleophiles that may be used in the displacement of trichloroacetimidates were undertaken. This project began as a new entry into the synthesis of $1,1^{\prime}$-diarylethane systems, as $1,1^{\prime}$-diarylethanes are an important functionality in many biologically active molecules ${ }^{9}$ like those shown in Figure $1 .{ }^{10}$ Typically these systems are accessed by hydrogenation of an alkene, ${ }^{11}$ displacement of a leaving group (like a halide, ${ }^{12}$ ammonium salt ${ }^{13}$ or ether ${ }^{14}$ ) or through Friedel-Crafts processes, ${ }^{15}$ however displacement of the imidate may also provide access to these systems if a compatible alkylmetal nucleophile could be invoked.

A survey of the literature suggested that trimethylaluminum may be a good candidate for this study as this reagent is readily available. Additionally, trimethylaluminum addition reactions are tolerant of and accelerated by exogenous Lewis acids, ${ }^{16}$ which are also known to facilitate trichloroacetimidate displacements. Trimethylaluminum has been used as a source of methyl anion previously to displace halides, ${ }^{17}$ sulfonates, ${ }^{17 b, 18}$ and Meldrum's acid derivatives. ${ }^{19}$ This reagent has also been shown to open benzylic epoxides ${ }^{20}$ and benzylic sulfites, ${ }^{21}$ with the addition of the new methyl group occurring preferentially at the benzylic position in these cyclic systems. Yields in these displacements and ring openings tend to be highest when the leaving group is tertiary or benzylic, ${ }^{17 c, 22}$ and most studies indicate that the trimethylaluminum is adding to carbocations that are formed in situ. This mechanism is supported by the rearrangement of some tertiary allylic chlorides during the addition reaction. ${ }^{23}$

Initially, the reaction of trimethylaluminum with diphenylmethyl trichloroacetimidate $\mathbf{6 a}$ was studied (Table 1). Solvent effects were observed to be prevalent in the substitution, as only DCM provided 1,1-diphenylethane 7a in appreciable yield at room temperature (entries 1-5). More polar solvents like $\mathrm{Et}_{2} \mathrm{O}$, THF and $\mathrm{MeCN}$ may coordinate to the trimethylaluminum and lower reactivity, reducing conversion. Alternatively, the less polar toluene may disfavor formation of the diphenylmethyl carbocation, which may be necessary for product formation. Heating the reaction in toluene did provide some product, however no conversion was observed in refluxing THF. In order to improve the yield, the use of Lewis acid promoters was explored. Initially TMSOTf and $\mathrm{BF}_{3} \cdot \mathrm{OEt}_{2}$ were evaluated, as these Lewis acids are commonly employed with trichloroacetimidates. ${ }^{16 a}, 16 \mathrm{c}, 16 \mathrm{~d}$ With TMSOTf, however, the major product was the rearranged trichloroacetamide 8a, and this product was also a significant in the reaction with $\mathrm{BF}_{3} \cdot \mathrm{OEt}_{2}$. This product has recently been observed to occur when trichloroacetimidate $\mathbf{6 a}$ is treated with a Lewis acid. ${ }^{24}$ A number of other Lewis acids were then screened for their ability to facilitate the substitution reaction (entries 1115 ) at the expense of the rearrangement. From these results aluminum trichloride emerged as the most promising promoter, providing the desired product $\mathbf{7 a}$ in a $91 \%$ yield (entry 11). Omission of the trimethylaluminum was also explored, with mainly rearrangement of the 
imidate to the acetamide being observed under these conditions (entry 16), as has been described previously. ${ }^{24}$

The scope of the displacement with respect to trichloroacetimidate was then investigated (Table 2). Initially diarylmethane-type trichloroacetimidates bearing different functionality on the aromatic rings were evaluated. Alkyl, aryl and methoxy functionalized aromatic substrates all provided the corresponding methylated products in good yield (entries 2-5). In the case of the methoxy containing imidate $6 \mathbf{e}$ some rearrangement (28\%) to the corresponding trichloroacetamide was observed. In the other examples in Table 2 only trace amounts of the rearranged product was observed, evidently this rearrangement is most competitive in highly activated electron rich systems. The conditions were also compatible with aryl halides (entries 6-8). Incorporation of stronger electron withdrawing groups on one of the aromatic rings was also tolerated in the diarylmethyl imidate substitutions, with nitro and trifluoromethyl groups not troubling the displacement (entries 9 and 10). These results are similar to Friedel-Crafts reactions that have been reported with electron-poor trichloroacetimidates, which also proceed in good yield. ${ }^{25}$ Larger bicyclic aromatic systems like naphthalene and benzothiophene were incorporated without issue (entries 11-14). The synthesis of the benzothiophene containing $1,1^{\prime}$-diarylethane $\mathbf{7 n}$ is notable, as this compound is an intermediate in the synthesis of benzothiophene $\mathbf{5}$, a compound designed to treat insomnia. ${ }^{26}$

With the success of diarylmethyl trichloroacetimidates in the methylation reaction, attention was focused on less activated substrates. Additionally, the diarylmethanes cannot undergo competing elimination reactions, so some systems that could be susceptible to elimination were evaluated (Table 2, entries 15-19). Most of these substrates performed well, including the hindered substrate 6q. Substitution with imidate $\mathbf{6 q}$ had to be started at a lower temperature, as when the reaction was started at $0{ }^{\circ} \mathrm{C}$ the alkene derived from elimination of the imidate was the major product. Primary benzylic trichloroacetimidates (Table 2, entries 20-26) were more reactive and did not require the addition of aluminum trichloride to provide the ethyl-substituted aromatics in useful yields. In addition, many of the primary imidate substitutions were initiated at low temperatures to avoid decomposition and/or elimination. In the case of 4-nitrobenzyl trichloroacetimidate no reaction occurred, with only starting material being recovered. Attempts to force substitution of imidate $\mathbf{6 z}$ by heating the reaction in DCE at reflux also gave no reaction. The aliphatic imidate $6 \mathbf{a a}$ was also unreactive and only returned starting material, highlighting the requirement for benzylic activation of the imidate.

Tertiary benzylic trichloroacetimidates and allylic trichloroacetimidates were also evaluated as substrates (Scheme 1). Displacement of the tertiary benzylic trichloroacetimidate 9 (prepared from the known alcohol ${ }^{27}$ ) may provide an expeditious route to analogs of physostigmine. ${ }^{28}$ Attempts to convert imidate 9 to the desired methyl compound were unsuccessful, as only the corresponding chloride $\mathbf{1 0}$ was obtained. Although chloride displacement of trichloroacetimidates is known with $\mathrm{HCl}^{29}$ the formation of chloride $\mathbf{1 0}$ was unexpected as trimethylaluminum typically is a better nucleophile than the chloride ion. Attempts to achieve methyl substitution using other Lewis acid promoters with less nucleophilic counterions (TMSOTf, $\mathrm{Zn}(\mathrm{OTf})_{2}$ ) provided starting material at room 
temperature or below. Alternatively, heating chloride $\mathbf{1 0}$ with two equiv of trimethylaluminum in refluxing DCE only returned starting material, and no methylation product was detected. While the displacement of tertiary chlorides with trimethylaluminum has been reported, the reactions are slower in the presence of Lewis basic functional groups like amides. ${ }^{17 \mathrm{c}}$ This result was rationalized by complexation of the trimethylaluminum to the carbamate leading to a slower substitution reaction. In the more complex system $\mathbf{9}$ when the trimethylaluminum is complexed with the carbamate, ester and sulfonamide, displacement by the methyl group may be decelerated leading to the formation of chloride $\mathbf{1 0}$. The formation of chloride $\mathbf{1 0}$ also suggests that the corresponding chlorides may be intermediates in the methyl displacement reaction. To investigate this possibility, chlorodiphenylmethane was treated with trimethylaluminum (DCM room temp, $0{ }^{\circ} \mathrm{C}-\mathrm{rt}$ ) which resulted in the formation of $1,1^{\prime}$-diphenylethane $7 \mathbf{a}$ in $65 \%$ yield, supporting the possibility that if the chloride was formed it would be rapidly displaced by trimethylaluminum. Attempts to isolate chlorodiphenylmethane by treating imidate $\mathbf{6 a}$ with $\mathrm{AlCl}_{3}$ in DCM gave predominantly the rearrangement product, acetamide $\mathbf{8 a}(60 \%$ yield, Table 1, entry 16). Only trace amounts of chlorodiphenylmethane in the crude ${ }^{1} \mathrm{H}$ NMR. We are therefore unable to unambiguously assess whether the corresponding chloride is an intermediate in the transformation, and it is also unclear why chloride displacement is favored over imidate rearrangement or elimination for imidate $\mathbf{9}$.

Evaluation of the imidate of allylic alcohol $\mathbf{1 1}$ was stymied by the instability of the trichloroacetimidate, which readily decomposed. Adopting the procedure of Dalla and coworkers, ${ }^{6 \mathrm{~b}}$ where the imidate was formed and used without purification in the alkylation reaction, gave alkene $\mathbf{1 2}$ as the sole product of the reaction in 63\% yield over two steps. Interestingly, none of the direct substitution product 13 was detected in the crude ${ }^{1} \mathrm{H}$ NMR.

As shown in Table 2, imidates that are precursors to relatively stable carbocations tend to provide higher yields in the substitution reaction. This is consistent with the reaction proceeding through an $\mathrm{S}_{\mathrm{N}} 1$-type pathway, where the imidate ionizes and the resulting cation is trapped by the trimethylaluminum. Additionally, the formation of the more hindered allylic substitution product $\mathbf{1 2}$ (Scheme 1) indicates that sterics is not a controlling factor in the substitution reaction, which is also consistent with an $\mathrm{S}_{\mathrm{N}} 1$ pathway. To further test this hypothesis the enantioenriched imidate $(S)-\mathbf{1 5}$ was prepared from the known aminoalcohol ${ }^{30}$ and subjected to the reaction conditions, providing the product $\mathbf{1 6}$ in $49 \%$ yield (Scheme 2). Analysis of the reaction product by chiral HPLC showed a racemic mixture, providing further support for an $\mathrm{S}_{\mathrm{N}} 1$-type mechanism being operative.

The use of triethylaluminum as the nucleophile in the imidate substitution was also briefly evaluated. In contrast to trimethylaluminum (Table 1, entry 2), treatment of imidate $6 \mathbf{a}$ with triethylaluminum provided the rearranged acetamide $7 \mathbf{a}(60 \%)$ as the major product of the reaction (Scheme 3). While a trace amount of the ethyl addition product $\mathbf{1 7}$ was observed, it was contaminated with the reduction product diphenylmethane $\mathbf{1 8}$, which was difficult to separate. This product may arise from $\beta$-hydride delivery, as has been observed in the reaction of triethylaluminum with benzylic halides. ${ }^{17 a}$ Triethylaluminum has also been previously reported to reductively open epoxides ${ }^{31}$ and reduce activated alcohols to alkanes $^{32}$ through a similar mechanism. Given the predominant rearrangement product, the 
added complexity of the competitive reduction, and the difficulty in separating the ethyl addition and reduction products, the use of triethylaluminum was not explored further.

The Lewis acid promoted addition of trimethylaluminum to trichloroacetimidates provides a useful method for the transformation of alcohols to the corresponding methyl analogs. This work represents the first conditions for the displacement of trichloroacetimidates with an alkylmetal reagent. The transformation most likely proceeds through cationic intermediates, and therefore performs best in benzylic or allylic systems. Increasing steric demands around the site of rearrangement was detrimental to the yield of the transformation. This reaction is especially useful in the synthesis of $1,1^{\prime}$-diarylethanes and related systems, which are important functionality in drug discovery. The reactivity of trichloroacetimidates with other organometallic reagents (specifically organozinc and organocopper reagents) is now being explored, and the results of these studies will be reported in due course.

\section{Experimental Section}

\section{General Procedure for Trichloroacetimidate Synthesis}

A flame dried flask was charged with the alcohol starting material (1 equiv) under argon. Dry DCM was then added to form a $0.5 \mathrm{M}$ solution, and the flask was cooled to $0{ }^{\circ} \mathrm{C}$. 1,8 Diazabicyclo[5.4.0] undec-7-ene ( 0.2 equiv) was then added to the solution, followed by trichloroacetonitrile (1.5 equiv). The reaction mixture was monitored by TLC until no more alcohol starting material was detected. The reaction mixture was then concentrated in vacuo, and the residue was purified by silica gel column chromatography using the listed solvent system to provide the desired trichloroacetimidates. Imidates $6 \mathbf{b}, \mathbf{6 c}, \mathbf{6 d}, \mathbf{6 e}, \mathbf{6 f}, \mathbf{6 g}, \mathbf{6 h}, \mathbf{6 j}$, 61, 6m, 6n, 6o, 6p, 6q, 6r, 6s, 6t, 6w, 6x, 6y, 6z, 6aa, 9 and 15 were synthesized from the corresponding alcohols using the general procedure. Imidates $6 \mathbf{a},{ }^{1 \mathrm{c}} \mathbf{6 i},{ }^{24} \mathbf{6 k},{ }^{33} \mathbf{6 u},{ }^{34}$ and $\mathbf{6 v}$, 35 were synthesized according to literature protocols.

(4-Methylphenyl)(phenyl)methyl 2,2,2-trichloroacetimidate (6b): Purified by silica gel chromatography (10\% ethyl acetate/1\% triethylamine/89\% hexanes). Viscous oil (1.17 g, 87\%). IR (DCM) 3340, 1662, 1286, 1066, $\left.998 \mathrm{~cm}^{-1 ;}{ }_{1}^{1} \mathrm{H} \mathrm{NMR} \mathrm{(400} \mathrm{MHz,} \mathrm{CDCl}_{3}\right) \delta 8.39$ (s, 1H), 7.43-7.41 (m, 2H), 7.36- $7.25(\mathrm{~m}, 5 \mathrm{H}), 7.15(\mathrm{~d}, J=7.9 \mathrm{~Hz}, 2 \mathrm{H}), 6.91(\mathrm{~s}, 1 \mathrm{H}), 2.32(\mathrm{~s}$, $3 \mathrm{H}) ;{ }^{13} \mathrm{C}\left\{{ }^{1} \mathrm{H}\right\} \mathrm{NMR}\left(100 \mathrm{MHz} \mathrm{CDCl}_{3}\right) \delta 161.3,139.9,137.8,136.8,129.2,128.4,127.9$, 127.0, 126.8, 91.6, 81.3, 21.6. Anal. calcd for $\mathrm{C}_{16} \mathrm{H}_{14} \mathrm{Cl}_{3} \mathrm{NO}$ : C, 56.09; H, 4.12; N, 4.09. Found: C, 56.32; H, 3.84; N, 4.44.

(2-Methylphenyl)(phenyl)methyl 2,2,2-trichloroacetimidate (6c): Purified by silica gel chromatography (5\% ethyl acetate/1\% triethylamine/94\% hexanes); Clear oil (2.38 g, 92\%); TLC $R_{\mathrm{f}}=0.59$ (10\% ethyl acetate/90\% hexanes); IR (DCM) 3339, 3053, 3032, 2983, 1664, 1286, 1265, 1075, 913, $741 \mathrm{~cm}^{-1 ;}{ }_{1} \mathrm{H}$ NMR (400 MHz, CDCl $) \delta 8.39$ (brs, 1H), 7.46-7.43 $(\mathrm{m}, 1 \mathrm{H}), 7.38-7.28(\mathrm{~m}, 5 \mathrm{H}), 7.24-7.16(\mathrm{~m}, 3 \mathrm{H}), 7.12(\mathrm{~s}, 1 \mathrm{H}), 2.37(\mathrm{~s}, 3 \mathrm{H}) ;{ }^{13} \mathrm{C}\left\{{ }^{1} \mathrm{H}\right\} \mathrm{NMR}$ $\left(100 \mathrm{MHz}, \mathrm{CDCl}_{3}\right) \delta 161.3,138.9,137.5,136.2,130.5,128.4,128.1,128.0,127.3,127.2$, 126.1, 91.6, 79.0, 19.4. Anal. calcd for $\mathrm{C}_{16} \mathrm{H}_{14} \mathrm{Cl}_{3} \mathrm{NO}$ : C, 56.09; H, 4.12; N, 4.09. Found: $\mathrm{C}$, $56.00 ; \mathrm{H}, 3.96 ; \mathrm{N}, 4.44$. 
[1,1'-Biphenyl]-4-yl](phenyl)methyl 2,2,2-trichloroacetimidate (6d): Purified by silica gel chromatography (1\% triethylamine/99\% hexanes); White solid (0.35 g, 75\%); IR (DCM) 3339, 3052, 1664, 1493, 1265, 1075, 984, 798, 739, $703 \mathrm{~cm}^{-1 ;}{ }_{1} \mathrm{H}$ NMR (300 MHz, $\mathrm{CDCl}_{3}$ ) $\delta 8.42$ (brs, $1 \mathrm{H}), 7.58-7.29(\mathrm{~m}, 14 \mathrm{H}), 6.97(\mathrm{~s}, 1 \mathrm{H}) .{ }^{13} \mathrm{C}\left\{{ }^{1} \mathrm{H}\right\}$ NMR $\left(100 \mathrm{MHz}, \mathrm{CDCl}_{3}\right) \delta$ 161.4, 141.0, 140.7, 139.8, 138.8, 128.9, 128.6, 128.2, 127.5, 127.4, 127.2, 127.0, 91.7, 81.3 (one aromatic resonance was unresolved). Anal. calcd for $\mathrm{C}_{21} \mathrm{H}_{16} \mathrm{Cl}_{3} \mathrm{NO}: \mathrm{C}, 62.32 ; \mathrm{H}, 3.99$; N, 3.46. Found: C, 62.50; H, 3.98; N, 3.59.

(4-Methoxyphenyl)(phenyl)methyl 2,2,2-trichloroacetimidate (6e): Purified by silica gel chromatography ( $1 \%$ ethyl acetate/1\% triethylamine/98\% hexanes); Yellow viscous oil (1.32 g, 92\%); IR (DCM) 3335, 1663, 1609, 1512, 1268, 1054, 915, $739 \mathrm{~cm}^{-1 ; 1} \mathrm{H}$ NMR (400 $\left.\mathrm{MHz}, \mathrm{CDCl}_{3}\right) \delta 8.38(\mathrm{~s}, 1 \mathrm{H}), 7.42-7.25(\mathrm{~m}, 7 \mathrm{H}), 6.90-6.86(\mathrm{~m}, 3 \mathrm{H}), 3.79(\mathrm{~s}, 3 \mathrm{H}) ;{ }^{13} \mathrm{C}\left\{{ }^{1} \mathrm{H}\right\}$ NMR $\left(100 \mathrm{MHz}, \mathrm{CDCl}_{3}\right) \delta 161.4,159.4,140.0,131.9,128.6,128.5,127.9,126.8,113.9$, 91.7, 81.2, 55.3. Anal. calcd for $\mathrm{C}_{16} \mathrm{H}_{14} \mathrm{Cl}_{3} \mathrm{NO}_{2}$ : C, 53.58; H, 3.93; N, 3.91. Found: C, $53.83 ; \mathrm{H}, 3.67 ; \mathrm{N}, 4.15$.

(3-Chlorophenyl)(phenyl)methyl 2,2,2-trichloroacetimidate (6f): Purified by silica gel chromatography (5\% ethyl acetate/1\% triethylamine/94\% hexanes); Viscous oil (1.23 g, $78 \%$ ); TLC $\mathrm{R}_{\mathrm{f}}=0.65$ (10\% ethyl acetate/90\% hexanes); IR (DCM) 3341, 1664, 1354, 1266, 1007, 791, 720, $701 \mathrm{~cm}^{-1 ;}{ }_{1} \mathrm{H}$ NMR (400 MHz, CDCl $) \delta 8.44$ (brs, $1 \mathrm{H}$ ), 7.41 (d, $J=8.9$ $\mathrm{Hz}, 3 \mathrm{H}), 7.37-7.21(\mathrm{~m}, 6 \mathrm{H}), 6.90(\mathrm{~s}, 1 \mathrm{H}) ;{ }^{13} \mathrm{C}\left\{{ }^{1} \mathrm{H}\right\} \mathrm{NMR}\left(100 \mathrm{MHz}, \mathrm{CDCl}_{3}\right) \delta 161.2,141.9$, 139.1, 134.5, 129.9, 128.7, 128.4, 128.3, 127.1, 127.0, 125.1, 91.4, 80.6. Anal. Calcd for $\mathrm{C}_{15} \mathrm{H}_{11} \mathrm{Cl}_{4} \mathrm{NO}: \mathrm{C}, 49.62 ; \mathrm{H}, 3.05 ; \mathrm{N}, 3.86$. Found: $\mathrm{C}, 49.85 ; \mathrm{H}, 3.25 ; \mathrm{N}, 4.00$.

(4-Bromophenyl)(phenyl)methyl 2,2,2-trichloroacetimidate (6g): Purified by silica gel chromatography (5\% ethyl acetate/1\% triethylamine/94\% hexanes); White solid ( $2.08 \mathrm{~g}$, 90\%); TLC $\mathrm{R}_{\mathrm{f}}=0.59$ (10\% ethyl acetate/90\% hexanes); IR (DCM) 3054, 2986, 2305, 1667, 1487, 1421, 1265, $705 \mathrm{~cm}^{-1 ;}{ }_{1} \mathrm{H}$ NMR $\left(400 \mathrm{MHz}, \mathrm{CDCl}_{3}\right) \delta 8.43$ (brs, $\left.1 \mathrm{H}\right), 7.48$ (d, $J=8.5$ $\mathrm{Hz}, 2 \mathrm{H}), 7.34-7.29(\mathrm{~m}, 7 \mathrm{H}), 6.89(\mathrm{~s}, 1 \mathrm{H}) ;{ }^{13} \mathrm{C}\left\{{ }^{1} \mathrm{H}\right\} \mathrm{NMR}\left(100 \mathrm{MHz}, \mathrm{CDCl}_{3}\right) \delta$ 161.2, 139.2, 138.8, 131.7, 128.7, 128.6, 128.3, 126.9, 122.1, 91.4, 80.7. Anal. Calcd for $\mathrm{C}_{15} \mathrm{H}_{11} \mathrm{BrCl}_{3} \mathrm{NO}: \mathrm{C}, 44.21 ; \mathrm{H}, 2.72 ; \mathrm{N}, 3.44$. Found: $\mathrm{C}, 44.18 ; \mathrm{H}, 2.98 ; \mathrm{N}, 3.76$.

(3-Fluorophenyl)(phenyl)methyl 2,2,2-trichloroacetimidate (6h): Purified by silica gel chromatography (5\% ethyl acetate/1\% triethylamine/94\% hexanes); Clear oil (0.97 g, 56\%); TLC $\mathrm{R}_{\mathrm{f}}=0.61$ (10\% ethyl acetate/90\% hexanes); IR (DCM) 3054, 1666, 1076, 999, 797, $735 \mathrm{~cm}^{-1 ;}{ }_{1} \mathrm{H}$ NMR $\left(400 \mathrm{MHz}, \mathrm{CDCl}_{3}\right) \delta 8.44$ (brs, $\left.1 \mathrm{H}\right), 7.43-7.41(\mathrm{~m}, 2 \mathrm{H}), 7.38-7.28(\mathrm{~m}$, $4 \mathrm{H}), 7.21-7.13(\mathrm{~m}, 2 \mathrm{H}), 7.00-6.95(\mathrm{~m}, 1 \mathrm{H}), 6.92(\mathrm{~s}, 1 \mathrm{H}) ;{ }^{13} \mathrm{C}\left\{{ }^{1} \mathrm{H}\right\} \mathrm{NMR}\left(100 \mathrm{MHz}, \mathrm{CDCl}_{3}\right)$ 162.8 (d, $J=245.1 \mathrm{~Hz}), 161.2,142.3,142.3,139.1,130.1,130.0,128.6,128.3,127.0,122.5$, $114.9(\mathrm{~d}, J=21.0 \mathrm{~Hz}), 113.9(\mathrm{~d}, J=22.2 \mathrm{~Hz}), 91.4,80.6$. Anal. Calcd for $\mathrm{C}_{15} \mathrm{H}_{11} \mathrm{Cl}_{3} \mathrm{FNO}$ : C, 51.98; H, 3.20; N, 4.04. Found: C, 52.06; H, 3.02; N, 4.06.

[3,5-Bis(trifluoromethyl)phenyl](phenyl)methyl 2,2,2-trichloroacetimidate (6j): Purified by silica gel chromatography (10\% ethyl acetate/1\% triethylamine/89\% hexanes); Off-white solid (1.25 g, 89\%); TLC R $\mathrm{R}_{\mathrm{f}}=0.52$ (10\% ethyl acetate/90\% hexanes); IR (DCM) 3342, 1668, 1625, 1379, 963, $798 \mathrm{~cm}^{-1 ; 1} \mathrm{H}$ NMR (400 MHz, $\left.\mathrm{CDCl}_{3}\right) \delta 8.52(\mathrm{~s}, 1 \mathrm{H}), 7.89(\mathrm{~s}, 2 \mathrm{H})$, $7.82(1 \mathrm{H}), 7.42-7.36(\mathrm{~m}, 5 \mathrm{H}), 7.03(\mathrm{~s}, 1 \mathrm{H}) ;{ }^{13} \mathrm{C}\left\{{ }^{1} \mathrm{H}\right\} \mathrm{NMR}\left(100 \mathrm{MHz}, \mathrm{CDCl}_{3}\right) \delta 160.9$, 
$142.5,137.9,132.0$ (q, $J=45.0 \mathrm{~Hz}), 129.0,128.9,123.2(\mathrm{q}, J=361.7 \mathrm{~Hz}), 122.1$ (hept, $J=$ $4.9 \mathrm{~Hz}$ ), 91.1, 79.0 (two aromatic resonances did not resolve). Anal. Calcd for $\mathrm{C}_{17} \mathrm{H}_{10} \mathrm{Cl}_{3} \mathrm{~F}_{6} \mathrm{NO}: \mathrm{C}, 43.95 ; \mathrm{H}, 2.17$; N, 3.01. Found: $\mathrm{C}, 43.83 ; \mathrm{H}, 2.07 ; \mathrm{N}, 3.14$.

(Naphthalen-1-yl)(phenyl)methyl 2,2,2-trichloroacetimidate (61): Purified by silica gel chromatography ( $1 \%$ triethylamine/99\% hexanes); Clear oil (1.84 g, 70\%); $\mathrm{TLC} \mathrm{R}_{\mathrm{f}}=0.61$ (10\% ethyl acetate/90\% hexanes); IR (DCM) 3055, 1665, 1265, 1074, 981, $735 \mathrm{~cm}^{-1 ; 1} \mathrm{H}$ NMR (300 MHz, $\left.\mathrm{CDCl}_{3}\right) \delta 8.47$ (brs, 1H), 8.08-8.05 (m, 1H), 7.88-7.83 (m, 1H), $7.70(\mathrm{~s}$, $1 \mathrm{H}), 7.61(\mathrm{~d}, J=7.0 \mathrm{~Hz}, 1 \mathrm{H}), 7.49-7.44(\mathrm{~m}, 5 \mathrm{H}), 7.37-7.29(\mathrm{~m}, 3 \mathrm{H}), 7.23(\mathrm{~s}, 1 \mathrm{H}) ;{ }^{13} \mathrm{C}\left\{{ }^{1} \mathrm{H}\right\}$ NMR $\left(100 \mathrm{MHz}, \mathrm{CDCl}_{3}\right) \delta 161.5,139.1,134.8,133.9,130.9,129.1,128.8,128.5,128.1$, 127.3, 126.4, 126.0, 125.8, 125.2, 124.0, 91.6, 79.2. Anal. Calcd for $\mathrm{C}_{19} \mathrm{H}_{14} \mathrm{Cl}_{3} \mathrm{NO}: \mathrm{C}$, 60.26; H, 3.73; N, 3.70. Found: C, 60.20; H, 3.85; N, 3.92.

(1-Benzothiophen-3-yl)(phenyl)methyl 2,2,2-trichloroacetimidate (6m): Purified by silica gel chromatography (5\% ethyl acetate/1\% triethylamine/94\% hexanes); Viscous oil (0.34 g, 61\%); IR (DCM) 3335, 1664, 1264, 702, 563, $484 \mathrm{~cm}^{-1 ;}{ }_{1} \mathrm{H}$ NMR (400 MHz, $\left.\mathrm{CDCl}_{3}\right) \delta 8.49(\mathrm{brs}, 1 \mathrm{H}), 7.86-7.84(\mathrm{~m}, 1 \mathrm{H}), 7.78-7.75(\mathrm{~m}, 1 \mathrm{H}), 7.52(\mathrm{~d}, J=7.0 \mathrm{~Hz}, 1 \mathrm{H})$, 7.40-7.32 (m, 7H), $7.25(\mathrm{~s}, 1 \mathrm{H}) ;{ }^{13} \mathrm{C}\left\{{ }^{1} \mathrm{H}\right\}$ NMR $\left(100 \mathrm{MHz}, \mathrm{CDCl}_{3}\right) \delta 161.4,140.7,138.2$, 137.3, 134.5, 128.6, 128.5, 127.3, 125.9, 124.7, 124.3, 122.9, 122.7, 91.6, 77.2. Anal. Calcd for $\mathrm{C}_{17} \mathrm{H}_{12} \mathrm{Cl}_{3} \mathrm{NOS}$ : C, 53.08; H, 3.14; N, 3.64. Found: C, 53.29; H, 2.92; N, 4.01 .

(1-Benzothiophen-3-yl)(4-fluorophenyl)methyl 2,2,2-trichloroacetimidate (6n): Purified by silica gel chromatography (5\% ethyl acetate/1\% triethylamine/94\% hexanes); Clear oil $(0.90 \mathrm{~g}, 71 \%)$; TLC $\mathrm{R}_{\mathrm{f}}=0.76$ (10\% ethyl acetate/90\% hexanes); IR (DCM) 3338, 3052, $1665,1606,1510,1265,1071 \mathrm{~cm}^{-1 ; 1} \mathrm{H}$ NMR $\left(300 \mathrm{MHz}, \mathrm{CDCl}_{3}\right) \delta 8.50(\mathrm{brs}, 1 \mathrm{H})$, 7.87-7.83 (m, 1H), 7.72-7.68 (m, 1H), 7.52-7.47 (m, 2H), 7.37 (d, $J=0.9 \mathrm{~Hz}, 1 \mathrm{H}), 7.35-7.31$ $(\mathrm{m}, 3 \mathrm{H}), 7.06$ (tt, $J=9.8,2.9 \mathrm{~Hz}, 2 \mathrm{H}) ;{ }^{13} \mathrm{C}\left\{{ }^{1} \mathrm{H}\right\} \mathrm{NMR}\left(100 \mathrm{MHz}, \mathrm{CDCl}_{3}\right) \delta 162.7(\mathrm{~d}, J=$ $246.3 \mathrm{~Hz}), 161.3,140.7,137.1,134.2,134.0,129.3$ (d, $J=6.7 \mathrm{~Hz}), 125.7,124.7,124.3$, 122.9, 122.6, $115.6(\mathrm{~d}, J=29.0 \mathrm{~Hz}), 91.5$, 77.4. Anal. Calcd for $\mathrm{C}_{17} \mathrm{H}_{11} \mathrm{Cl}_{3} \mathrm{FNOS}: \mathrm{C}$, 50.71; H, 2.75; N, 3.48. Found: C, 50.77; H, 2.70; N, 3.53.

1-(1,1'-Biphenyl]-4-yl)ethyl 2,2,2-trichloroacetimidate (6o): Purified by silica gel chromatography (5\% ethyl acetate/1\% triethylamine/94\% hexanes); Off white solid (1.06 g, $81 \%$ ); IR (DCM) 3333, 1661, 1661, 798, 765, 702, $650 \mathrm{~cm}^{-1 ; 1} \mathrm{H} \mathrm{NMR} \mathrm{(400} \mathrm{MHz,} \mathrm{CDCl}_{3}$ ) $\delta 8.33$ (brs, 1H), 7.60-7.57 (m, 4H), 7.49 (d, $J=8.2 \mathrm{~Hz}, 2 \mathrm{H}), 7.43(\mathrm{t}, J=7.3 \mathrm{~Hz}, 2 \mathrm{H})$, 7.36-7.32 (m, 1H), $6.03(\mathrm{q}, J=6.5 \mathrm{~Hz}, 1 \mathrm{H}), 1.69(\mathrm{~d}, J=6.6 \mathrm{~Hz}, 3 \mathrm{H}) ;{ }^{13} \mathrm{C}\left\{{ }^{1} \mathrm{H}\right\}$ NMR $(75$ $\left.\mathrm{MHz}, \mathrm{CDCl}_{3}\right) \delta 161.7,140.9,140.8,140.4,128.8,127.4,127.3,127.1,126.3,91.8,77.0$, 22.1. Anal. Calcd for $\mathrm{C}_{16} \mathrm{H}_{14} \mathrm{Cl}_{3} \mathrm{NO}$ : C, 56.09; H, 4.12; N, 4.09. Found: C, 56.00; H, 3.96; $\mathrm{N}, 4.44$.

1-(1,1'-Biphenyl]-4-yl)pentyl 2,2,2-trichloroacetimidate (6p): Purified by silica gel chromatography (5\% ethyl acetate/1\% triethylamine/94\% hexanes); White solid $(0.71 \mathrm{~g}$, $60 \%$ ); TLC $\mathrm{R}_{\mathrm{f}}=0.68$ (10\% ethyl acetate/90\% hexanes); IR (DCM) 3339, 2954, 2927, 2859, $1661,1300 \mathrm{~cm}^{-1 ; 1} \mathrm{H}$ NMR (400 MHz, $\left.\mathrm{CDCl}_{3}\right) \delta 8.27$ (brs, $\left.1 \mathrm{H}\right), 7.57$ (d, $\left.J=8.2 \mathrm{~Hz}, 4 \mathrm{H}\right)$, 7.47-7.32 (m, 4H), 7.34-7.32 (m, 1H), 5.87-5.84 (m, 1H), 2.12-2.03 (m, 1H), 1.92-1.84 (m, $1 \mathrm{H}), 1.51-1.34(\mathrm{~m}, 4 \mathrm{H}), 0.90(\mathrm{t}, J=7.2 \mathrm{~Hz}, 3 \mathrm{H}) ;{ }^{13} \mathrm{C}\left\{{ }^{1} \mathrm{H}\right\} \mathrm{NMR}\left(100 \mathrm{MHz}, \mathrm{CDCl}_{3}\right) \delta$ 
161.7, 140.7, 140.7, 139.5, 128.7, 127.1, 127.1, 126.5, 91.8, 80.8, 36.6, 27.6, 22.4, 14.0 (one aromatic resonance was not resolved). Anal. Calcd for $\mathrm{C}_{19} \mathrm{H}_{20} \mathrm{Cl}_{3} \mathrm{NO}: \mathrm{C}, 59.32 ; \mathrm{H}, 5.24 ; \mathrm{N}$, 3.64. Found: C, 59.58; H, 5.31; N, 3.45.

1-(1,1'-Biphenyl]-4-yl)-2-methylpropyl 2,2,2-trichloroacetimidate (6q): Purified by silica gel chromatography (10\% ethyl acetate/1\% triethylamine/89\% hexanes); White solid (2.00 $\mathrm{g}, 66 \%$ ), TLC $\mathrm{R}_{\mathrm{f}}=0.42$ (10\% ethyl acetate/90\% hexanes); IR (DCM) 3339, 2967, 1661, 1487, 1300, 1058, $990 \mathrm{~cm}^{-1 ; 1} \mathrm{H}$ NMR (400 MHz, $\left.\mathrm{CDCl}_{3}\right) \delta 8.16(\mathrm{~s}, 1 \mathrm{H}), 7.53-7.49(\mathrm{~m}, 4 \mathrm{H})$, 7.37-7.34 (m, 4H), 7.28-7.26 (m, 1H), $5.52(\mathrm{~d}, J=7.1 \mathrm{~Hz}, 1 \mathrm{H}), 2.16$ (octet, $J=6.8 \mathrm{~Hz}, 1 \mathrm{H}$ ), $1.02(\mathrm{~d}, J=6.6 \mathrm{~Hz}, 3 \mathrm{H}), 0.86(\mathrm{~d}, J=6.8 \mathrm{~Hz}, 3 \mathrm{H}) ;{ }^{13} \mathrm{C}\left\{{ }^{1} \mathrm{H}\right\} \mathrm{NMR}\left(100 \mathrm{MHz}, \mathrm{CDCl}_{3}\right) \delta$ 161.8, 140.8, 140.7, 138.2, 128.8, 127.4, 127.3, 127.2, 126.9, 92.0, 85.6, 34.4, 19.0, 18.3. Anal. Calcd for $\mathrm{C}_{18} \mathrm{H}_{18} \mathrm{Cl}_{3} \mathrm{NO}$ : C, 58.32; H, 4.89; N, 3.78. Found: C, 58.50; H, 4.69; N, 3.90 .

1-(Naphthalen-2-yl)propyl 2,2,2-trichloroacetimidate (6r): Purified by silica gel chromatography ( $1 \%$ triethylamine/99\% hexanes); Yellow oil (1.39 g, $96 \%$ ); TLC Rf $=0.62$ (10\% ethyl acetate/90\% hexanes); IR (DCM) 3332, 2972, 1663, 1073, 831, 797, $704 \mathrm{~cm}$ ${ }^{-1 ;}{ }_{1} \mathrm{H}$ NMR $\left(300 \mathrm{MHz}, \mathrm{CDCl}_{3}\right.$ ) $\delta 8.26$ (brs, $\left.1 \mathrm{H}\right), 7.85-7.81$ (m, 4H), 7.52 (dd, $J=8.4,1.6$ $\mathrm{Hz}, 1 \mathrm{H}), 7.48-7.45(\mathrm{~m}, 2 \mathrm{H}), 5.92(\mathrm{~m}, 1 \mathrm{H}), 2.20-2.09(\mathrm{~m}, 1 \mathrm{H}), 2.05-1.94(\mathrm{~m}, 1 \mathrm{H}), 1.03(\mathrm{t}, J=$ $7.4 \mathrm{~Hz}, 3 \mathrm{H}) ;{ }^{13} \mathrm{C}\left\{{ }^{1} \mathrm{H}\right\} \mathrm{NMR}\left(100 \mathrm{MHz}, \mathrm{CDCl}_{3}\right) \delta 161.8,137.5,133.08,133.06,128.2$, 128.0, 127.7, 126.2, 126.0, 125.4, 124.0, 91.9, 82.3, 29.8, 10.0. Anal. Calcd for $\mathrm{C}_{15} \mathrm{H}_{14} \mathrm{Cl}_{3} \mathrm{NO}$ : C; 54.49; H, 4.27; N, 4.24. Found: C, 54.42; H, 4.36; N, 4.31.

1-(1-Benzothiophen-3-yl)propyl 2,2,2-trichloroacetimidate (6s): Purified by silica gel chromatography (5\% ethyl acetate/1\% triethylamine/94\% hexanes); Yellow viscous oil (2.38 g, 91\%); IR (DCM) 3340, 2972, 1664, 1428, 1082, 976, $760 \mathrm{~cm}^{-1 ;}{ }_{1}^{1} \mathrm{H} \mathrm{NMR}(300 \mathrm{MHz}$, $\left.\mathrm{CDCl}_{3}\right) \delta 8.32$ (brs, $\left.1 \mathrm{H}\right), 7.94-7.91(\mathrm{~m}, 1 \mathrm{H}), 7.85-7.82(\mathrm{~m}, 1 \mathrm{H}), 7.45(\mathrm{~s}, 1 \mathrm{H}), 7.37-7.30(\mathrm{~m}$, $2 \mathrm{H}), 6.24(\mathrm{t}, J=8.1 \mathrm{~Hz}, 1 \mathrm{H}), 2.30-2.02(\mathrm{~m}, 2 \mathrm{H}), 1.02(\mathrm{t}, J=9.8 \mathrm{~Hz}, 3 \mathrm{H}) ;{ }^{13} \mathrm{C}\left\{{ }^{1} \mathrm{H}\right\} \mathrm{NMR}$ $\left(100 \mathrm{MHz}, \mathrm{CDCl}_{3}\right) \delta 161.9,140.7,137.1,134.9,124.5,124.1,123.6,122.9,122.5,91.9$, 77.9, 28.1, 10.0. Anal. Calcd for $\mathrm{C}_{13} \mathrm{H}_{12} \mathrm{Cl}_{3}$ NOS: C, 46.38; H, 3.59; $\mathrm{N}, 4.16$. Found: $\mathrm{C}$, $46.03 ; \mathrm{H}, 3.60 ; \mathrm{N}, 3.95$.

3,4-Dimethoxybenzyl 2,2,2-trichloroacetimidate (6t): Purified by silica gel chromatography (30\% ethyl acetate/1\% triethylamine/69\% hexanes); Yellow oil (3.00 g, $90 \%$ ); TLC $\mathrm{R}_{\mathrm{f}}=0.28$ (20\% ethyl acetate/80\% hexanes); ${ }^{1} \mathrm{H}$ NMR $\left(400 \mathrm{MHz}, \mathrm{CDCl}_{3}\right) \delta$ 8.38 (brs, $1 \mathrm{H}), 7.01-6.98(\mathrm{~m}, 2 \mathrm{H}), 6.87(\mathrm{~d}, J=8.1 \mathrm{~Hz}, 1 \mathrm{H}), 5.29(\mathrm{~s}, 2 \mathrm{H}), 3.89(\mathrm{~s}, 6 \mathrm{H})$; ${ }^{13} \mathrm{C}\left\{{ }^{1} \mathrm{H}\right\} \mathrm{NMR}\left(100 \mathrm{MHz}, \mathrm{CDCl}_{3}\right) \delta 162.4,149.1,149.0,127.9,120.8,111.3,111.0,91.5$, 70.8, 55.85, 55.83. Anal. Calcd for $\mathrm{C}_{11} \mathrm{H}_{12} \mathrm{Cl}_{3} \mathrm{NO}_{3}$ : C, 42.27; H, 3.87; $\mathrm{N}, 4.48$. Found: $\mathrm{C}$, $42.39 ; \mathrm{H}, 3.59 ; \mathrm{N}, 4.59$.

1-(Naphthalen-1-yl)ethyl 2,2,2-trichloroacetimidate (6w): Purified by silica gel chromatography (10\% ethyl acetate/1\% triethylamine/89\% hexanes); Light brown oil (5.64 $\mathrm{g}, 85 \%)$; TLC Rf $=0.56$ (10\% ethyl acetate/90\% hexanes); IR (DCM) 3338, 1662, 1512, 1301, 1085, 774, $648 \mathrm{~cm}^{-1 ; 1} \mathrm{H}$ NMR (400 MHz, $\left.\mathrm{CDCl}_{3}\right) \delta 8.47$ (brs, $\left.1 \mathrm{H}\right), 8.04(\mathrm{~d}, J=8.0$ $\mathrm{Hz}, 1 \mathrm{H}), 7.87$ (t, $J=7.4 \mathrm{~Hz}, 2 \mathrm{H}), 7.62(\mathrm{~d}, J=8.0 \mathrm{~Hz}, 1 \mathrm{H}), 7.56-7.44(\mathrm{~m}, 3 \mathrm{H}), 5.77$ (s, 2H); ${ }^{13} \mathrm{C}\left\{{ }^{1} \mathrm{H}\right\}$ NMR $\left(75 \mathrm{MHz}, \mathrm{CDCl}_{3}\right) \delta 162.8,133.7,131.6,130.9,129.5,128.7,127.3,126.5$, 
126.0, 125.2, 123.7, 91.5, 69.5. Anal. Calcd for $\mathrm{C}_{13} \mathrm{H}_{10} \mathrm{Cl}_{3} \mathrm{NO}$ : C, 51.60; H, 3.33; N, 4.63.

Found: C, 51.83; H, 3.28; N, 4.77 .

1-(Naphthalen-2-yl)ethyl 2,2,2-trichloroacetimidate (6x): Purified by silica gel chromatography (10\% ethyl acetate/1\% triethylamine/89\% hexanes); White solid (1.89 g, $99 \%$ ), TLC $\mathrm{R}_{\mathrm{f}}=0.70$ (20\% ethyl acetate/80\% hexanes); IR (DCM) 3335, 3053, 1665, 1305, 1081, 817, $649 \mathrm{~cm}^{-1 ;}{ }_{1}^{1} \mathrm{H}$ NMR (400 MHz, $\left.\mathrm{CDCl}_{3}\right) \delta 8.42$ (brs, 1H), $7.90(\mathrm{~s}, 1 \mathrm{H}), 7.87-7.84$ $(\mathrm{m}, 3 \mathrm{H}), 7.54(\mathrm{dd}, J=8.4,1.4 \mathrm{~Hz}, 1 \mathrm{H}), 7.50-7.48(\mathrm{~m}, 2 \mathrm{H}), 5.50(\mathrm{~s}, 2 \mathrm{H}),{ }^{13} \mathrm{C}\left\{{ }^{1} \mathrm{H}\right\}$ NMR $(100$ $\left.\mathrm{MHz}, \mathrm{CDCl}_{3}\right) \delta 162.6,133.2,133.1,132.9,128.4,128.0,127.7,127.0,126.3,126.3,125.5$, 91.4, 70.9. Anal. Calcd for $\mathrm{C}_{13} \mathrm{H}_{10} \mathrm{Cl}_{3} \mathrm{NO}$ : C, 51.60; H, 3.33; N, 4.63. Found: C, 51.71; H, $3.52 ; \mathrm{N}, 4.84$.

(1-Benzothiophen-3-yl)methyl 2,2,2-trichloroacetimidate (6y): Purified by silica gel chromatography (10\% ethyl acetate/1\% triethylamine/89\% hexanes); Off white solid (1.92 $\mathrm{g}, 68 \%$ ); TLC $\mathrm{R}_{\mathrm{f}}=0.48$ (10\% ethyl acetate/90\% hexanes); IR (DCM) 3340, 3052, 2984, 2304, 1664, 1460, 1295, 1265, 1076, 799, 737, $\left.705 \mathrm{~cm}^{-1 ; 1} \mathrm{H} \mathrm{NMR} \mathrm{(400} \mathrm{MHz,} \mathrm{CDCl}_{3}\right) \delta$ 8.46 (brs, $1 \mathrm{H}), 7.88-7.86(\mathrm{~m}, 2 \mathrm{H}), 7.56(\mathrm{~s}, 1 \mathrm{H}), 7.43-7.36(\mathrm{~m}, 2 \mathrm{H}), 5.57(\mathrm{~s}, 2 \mathrm{H}) ;{ }^{13} \mathrm{C}\left\{{ }^{1} \mathrm{H}\right\}$ NMR (75 MHz, $\left.\mathrm{CDCl}_{3}\right) \delta 162.7,140.5,137.8,130.3,126.5,124.7,124.4,122.9,122.0$, 91.0, 65.3. Anal. Calcd for $\mathrm{C}_{11} \mathrm{H}_{8} \mathrm{Cl}_{3} \mathrm{NOS}$ : C, 42.81; H, 2.61; N, 4.54. Found: C, 43.17; H, $2.43 ; \mathrm{N}, 4.61$.

4-Nitrobenzyl 2,2,2-trichloroacetimidate (6z): Purified by silica gel chromatography (20\% ethyl acetate/1\% triethylamine/79\% hexanes); Yellow solid (3.75 g, 97\%); TLC $\mathrm{R}_{\mathrm{f}}=0.23$ (10\% ethyl acetate/90\% hexanes); ${ }^{1} \mathrm{H}$ NMR $\left(400 \mathrm{MHz}, \mathrm{CDCl}_{3}\right) \delta 8.50(\mathrm{~s}, 1 \mathrm{H}), 8.25(\mathrm{~d}, J=$ $8.7 \mathrm{~Hz}, 2 \mathrm{H}), 7.61(\mathrm{~d}, J=8.8 \mathrm{~Hz}, 2 \mathrm{H}), 5.45(\mathrm{~s}, 2 \mathrm{H}),{ }^{13} \mathrm{C}\left\{{ }^{1} \mathrm{H}\right\} \mathrm{NMR}\left(100 \mathrm{MHz}, \mathrm{CDCl}_{3}\right) \delta$ 162.1, 147.8, 142.7, 127.9, 123.8, 90.9, 69.1. Anal. Calcd for $\mathrm{C}_{9} \mathrm{H}_{7} \mathrm{Cl}_{3} \mathrm{~N}_{2} \mathrm{O}_{3}$ : C, 36.33; $\mathrm{H}$, 2.37; N, 9.42. Found: C, 36.27; H, 2.30; N, 9.48.

2,2-Diphenylethyl 2,2,2-trichloroacetimidate (6aa): Purified by silica gel chromatography (5\% ethyl acetate/1\% triethylamine/89\% hexanes); Clear oil (0.31 g, 87\%); IR (DCM) 3337, $1663,1081,798 \mathrm{~cm}^{-1}$; TLC $\mathrm{R}_{\mathrm{f}}=0.68$ (10\% ethyl acetate/90\% hexanes); ${ }^{1} \mathrm{H}$ NMR (400 $\left.\mathrm{MHz}, \mathrm{CDCl}_{3}\right) \delta 8.30(\mathrm{~s}, 1 \mathrm{H}), 7.32-7.20(\mathrm{~m}, 10 \mathrm{H}), 4.81(\mathrm{~d}, J=7.3 \mathrm{~Hz}, 2 \mathrm{H}), 4.54(\mathrm{t}, J=7.2$ $\mathrm{Hz}, 1 \mathrm{H}) ;{ }^{13} \mathrm{C}\left\{{ }^{1} \mathrm{H}\right\} \mathrm{NMR}\left(100 \mathrm{MHz}, \mathrm{CDCl}_{3}\right) \delta 162.8,141.0,128.5,128.4,126.8,91.3,71.6$, 49.5. Anal. Calcd for $\mathrm{C}_{16} \mathrm{H}_{14} \mathrm{Cl}_{3} \mathrm{NO}$ : C, 56.09; H, 4.12; N, 4.09. Found: C, 55.93; H, 3.92; $\mathrm{N}, 4.07$.

(2S,3aS,8aS)-dimethyl 8-(phenylsulfonyl)-3a-(2,2,2-trichloro-1-iminoethoxy)-3,3a,8,8a tetrahydropyrrolo[2,3-b] indole-1,2(2H)-dicarboxylate (9): Purified by silica gel chromatography ( $40 \%$ ethyl acetate/ $1 \%$ triethylamine/59\% hexanes); White foamy solid $(0.08 \mathrm{~g}, 60 \%)$; TLC $\mathrm{R}_{\mathrm{f}}=0.57$ (50\% ethyl acetate $/ 50 \%$ hexanes); $[a]_{D}{ }^{21}=+85.1(\mathrm{c}=1.6$, $\mathrm{CHCl}_{3}$ ); IR (DCM) 3434, 2090, $\left.1642 \mathrm{~cm}^{-1 ; 1} \mathrm{H} \mathrm{NMR} \mathrm{(400} \mathrm{MHz,} \mathrm{CDCl}_{3}, 50{ }^{\circ} \mathrm{C}\right) \delta 8.49(\mathrm{~s}$, $1 \mathrm{H}), 7.98(\mathrm{~d}, J=7.2 \mathrm{~Hz}, 2 \mathrm{H}), 7.54-7.44(\mathrm{~m}, 4 \mathrm{H}), 7.39-7.30$ (m, 2H), $7.07(\mathrm{td}, J=7.7,1.1$ $\mathrm{Hz}, 1 \mathrm{H}), 6.71$ (s, 1H), 4.83 (d, $J=9.0 \mathrm{~Hz}, 1 \mathrm{H}), 3.51$ (brs, 3H), 3.26 (s, 3H), 3.17 (d, $J=12.9$ $\mathrm{Hz}, 1 \mathrm{H}), 3.03(\mathrm{dd}, J=12.8,9.2 \mathrm{~Hz}, 1 \mathrm{H}) ;{ }^{13} \mathrm{C}\left\{{ }^{1} \mathrm{H}\right\} \mathrm{NMR}\left(100 \mathrm{MHz}, \mathrm{CDCl}_{3}, 50{ }^{\circ} \mathrm{C}\right) \delta 170.3$, 159.1, 154.4, 144.8, 144.4, 132.5, 131.5, 129.0, 127.7, 126.6, 125.5, 124.3, 116.3, 93.4, 
90.8, 81.6, 58.6, 52.7, 52.2, 39.3. Anal. Calcd for $\mathrm{C}_{22} \mathrm{H}_{20} \mathrm{Cl}_{3} \mathrm{~N}_{3} \mathrm{O}_{7} \mathrm{~S}: \mathrm{C}, 45.81 ; \mathrm{H}, 3.49 ; \mathrm{N}$, 7.28. Found: C, 45.95; H, 3.37; N, 7.31.

3-(N-Methylbenzenesulfonamido)-1S-(thiophen-2-yl)propyl 2,2,2-trichloroacetimidate (15): Purified by silica gel chromatography (20\% ethyl acetate/1\% triethylamine/79\% hexanes); Yellow oil $(0.25 \mathrm{~g}, 85 \%)$; TLC $\mathrm{R}_{\mathrm{f}}=0.62$ (40\% ethyl acetate/60\% hexanes); $[a]_{D}{ }^{25}$ $=+32.5(\mathrm{c}=0.4, \mathrm{DCM})$; IR $(\mathrm{DCM}) 3375,1693,1335,1163,1109,834 \mathrm{~cm}^{-1 ; 1} \mathrm{H} \mathrm{NMR}(400$ $\left.\mathrm{MHz}, \mathrm{CDCl}_{3}\right) \delta 8.46$ (brs, 1H), 7.81-7.79 (m, 2H), 7.63-7.52 (m, 3H), 7.31 (dd, $J=5.1,1.1$ $\mathrm{Hz}, 1 \mathrm{H}), 7.18(\mathrm{~d}, J=3.4 \mathrm{~Hz}, 1 \mathrm{H}), 7.01(\mathrm{q}, J=3.6 \mathrm{~Hz}, 1 \mathrm{H}), 6.28(\mathrm{q}, J=4.9 \mathrm{~Hz}, 1 \mathrm{H}), 3.21(\mathrm{t}$, $J=6.9 \mathrm{~Hz}, 2 \mathrm{H}), 2.81(\mathrm{~s}, 3 \mathrm{H}), 2.47-2.38(\mathrm{~m}, 1 \mathrm{H}), 2.32-2.24(\mathrm{~m}, 1 \mathrm{H}) ;{ }^{13} \mathrm{C}\left\{{ }^{1} \mathrm{H}\right\} \mathrm{NMR}$ $\left(100 \mathrm{MHz}, \mathrm{CDCl}_{3}\right) \delta 161.1,141.4,137.4,132.7,129.1,127.4,126.6,126.5,125.7,91.4$, 73.5, 46.8, 35.5, 35.3. Anal. Calcd for $\mathrm{C}_{16} \mathrm{H}_{17} \mathrm{Cl}_{3} \mathrm{~N}_{2} \mathrm{O}_{3} \mathrm{~S}_{2}$ : C, 42.16; H, 3.76; N, 6.15. Found: $\mathrm{C}, 42.16 ; \mathrm{H}, 3.82 ; \mathrm{N}, 6.12$. The enantiomeric purity of this material was verified by NMR using the chiral shift reagent Europium tris[3-(heptafluoropropylhydroxymethylene)(+)-camphorate] i.e. $\mathrm{Eu}(\mathrm{hfc})_{3}$. The addition of 0.8 equiv of $\mathrm{Eu}(\mathrm{hfc})_{3}$ gave separation of the imidate $\mathrm{NH}$ peak at $\sim 8.4 \mathrm{ppm}$ in the ${ }^{1} \mathrm{H}$ NMR (see supporting spectra for details).

\section{General Procedures for the Displacement of Trichloroacetimidates with Trimethylaluminum}

Method A-Aluminium trichloride (1 eq) was dissolved in dry DCM $(0.162 \mathrm{M})$ under argon in a flame dried flask. Trimethylaluminium (3 equiv, $2 \mathrm{M}$ in hexanes) was slowly added and the mixture was stirred for $5 \mathrm{~min}$. After cooling to $0{ }^{\circ} \mathrm{C}$ the trichloroacetimidate (1 equiv) was added. The reaction mixture was then allowed to warm to rt. After 15 min the reaction was quenched with $10 \%$ aq. $\mathrm{HCl}$. The mixture was poured into water and extracted with DCM (3x). The combined organic extracts were dried $\left(\mathrm{Na}_{2} \mathrm{SO}_{4}\right)$, filtered, and concentrated in vacuo. The residue was then purified by silica gel chromatography using the eluent listed for each example. Compounds 7a, 7b, 7c, 7d, 7e, 7f, 7g, 7h, 7i, 7j, 7k, 7l, 7m, $7 \mathbf{n}, 7 \mathbf{7}, 7 \mathbf{p}, 7 \mathbf{r}, 7 \mathrm{~s}, \mathbf{1 0}$, and 16 were obtained by this method.

Method B-Trimethylaluminium (3 equiv, 2M in hexanes) was suspended in dry DCM $(0.162 \mathrm{M})$ under argon in a flame dried flask. The temperature of the reaction was then cooled to the temperature noted in Table 2. The trichloroacetimidate was the added. The reaction was monitored by TLC, and quenched with the addition of $10 \%$ aq. $\mathrm{HCl}$ after the time listed in Table 2. The quenched mixture was poured into water and extracted with DCM (3x). The organic extracts were then dried using $\mathrm{Na}_{2} \mathrm{SO}_{4}$, filtered, and concentrated. The residue was purified by silica gel chromatography using the eluent listed for each example. Compounds $\mathbf{7 q}, \mathbf{7 t}, \mathbf{7 u}, \mathbf{7 v}, \mathbf{7 w}, \mathbf{7 x}, \mathbf{7 y}$ were obtained using this method.

1,1- Diphenylethane (7a) ${ }^{36}$ : Purified by silica gel chromatography (1\% ethyl acetate/99\% hexanes); Clear viscous oil (0.04 g, 91\%); ${ }^{1} \mathrm{H}$ NMR (400 MHz, $\left.\mathrm{CDCl}_{3}\right)$ \& 7.20-7.08 (m, $10 \mathrm{H}), 4.07(\mathrm{q}, J=7.2 \mathrm{~Hz}, 1 \mathrm{H}), 1.56(\mathrm{~d}, J=7.2 \mathrm{~Hz}, 3 \mathrm{H}) ;{ }^{13} \mathrm{C}\left\{{ }^{1} \mathrm{H}\right\} \mathrm{NMR}\left(100 \mathrm{MHz}, \mathrm{CDCl}_{3}\right)$ $\delta$ 146.4, 128.4, 127.7, 126.1, 44.8, 21.9.

1-Phenyl-1-(p-tolyl)ethane (7b) ${ }^{\mathbf{3 6}}$ : Purified by silica gel chromatography (1\% ethyl acetate/99\% hexanes). Clear oil ( $0.03 \mathrm{~g}, 65 \%)$; $\mathrm{TLC} \mathrm{R}_{\mathrm{f}}=0.74$ (100\% hexanes); ${ }^{1} \mathrm{H}$ NMR $\left(400 \mathrm{MHz}, \mathrm{CDCl}_{3}\right) \delta$ 7.29-7.07 (m, 9H), 4.12 (q, $\left.J=7.2 \mathrm{~Hz}, 1 \mathrm{H}\right), 2.3(\mathrm{~s}, 3 \mathrm{H}), 1.62$ (d, $J=$ 
$7.2 \mathrm{~Hz}, 3 \mathrm{H}) ;{ }^{13} \mathrm{C}\left\{{ }^{1} \mathrm{H}\right\} \mathrm{NMR}\left(100 \mathrm{MHz}, \mathrm{CDCl}_{3}\right) \delta 146.6,143.4,135.5,129.0,128.3,127.6$, $127.5,125.9,44.4,21.9,21.0$.

1-Phenyl-1-(o-tolyl)ethane (7c) ${ }^{11 c}$ : Purified by silica gel chromatography (100\% hexanes). Clear viscous oil $(0.08 \mathrm{~g}, 70 \%)$; TLC $\mathrm{R}_{\mathrm{f}}=0.83$ (10\% ethyl acetate/90\% hexanes); ${ }^{1} \mathrm{H}$ NMR $\left(400 \mathrm{MHz}, \mathrm{CDCl}_{3}\right) \delta 7.27-7.11(\mathrm{~m}, 9 \mathrm{H}), 4.30(\mathrm{q}, J=7.2 \mathrm{~Hz}, 1 \mathrm{H}), 2.22(\mathrm{~s}, 3 \mathrm{H}), 1.60,(\mathrm{~d}, J=$ $7.2 \mathrm{~Hz}, 3 \mathrm{H}) ;{ }^{13} \mathrm{C}\left\{{ }^{1} \mathrm{H}\right\} \mathrm{NMR}\left(75 \mathrm{MHz}, \mathrm{CDCl}_{3}\right) \delta 146.3,144.0,136.2,130.5,128.4,127.8$, $126.8,126.2,126.1,125.9,41.1,22.2,19.8$.

4-(1-Phenylethyl)biphenyl (7d) ${ }^{37}$ : Purified by silica gel chromatography (1\% ethyl acetate/99\% hexanes); Clear viscous oil $(0.04 \mathrm{~g}, 91 \%)$; TLC $\mathrm{R}_{\mathrm{f}}=0.69$ (10\% ethyl acetate/90\% hexanes); ${ }^{1} \mathrm{H}$ NMR $\left(400 \mathrm{MHz}, \mathrm{CDCl}_{3}\right) \delta 7.55(\mathrm{dd}, J=8.4,1.4 \mathrm{~Hz}, 2 \mathrm{H})$, 7.51-7.49 (m, 2H), $7.40(\mathrm{t}, J=7.3 \mathrm{~Hz}, 2 \mathrm{H}), 7.32-7.16(\mathrm{~m}, 8 \mathrm{H}), 4.18(\mathrm{q}, J=7.2 \mathrm{~Hz}, 1 \mathrm{H}), 1.67$ $(\mathrm{d}, J=7.2 \mathrm{~Hz}, 3 \mathrm{H}) ;{ }^{13} \mathrm{C}\left\{{ }^{1} \mathrm{H}\right\} \mathrm{NMR}\left(100 \mathrm{MHz}, \mathrm{CDCl}_{3}\right) \delta 146.2,145.5,141.0,139.0,128.7$, 128.4, 128.0, 127.6, 127.1, 127.0, 127.0, 126.1, 44.5, 21.9.

4-(1-Phenylethyl)anisole (7e) ${ }^{38}$ : Purified by silica gel chromatography (1\% ethyl acetate/99\% hexanes); Clear viscous oil (0.03 g, 64\%); TLC $\mathrm{R}_{\mathrm{f}}=0.37$ (10\% ethyl acetate/90\% hexanes); ${ }^{1} \mathrm{H}$ NMR $\left(400 \mathrm{MHz}, \mathrm{CDCl}_{3}\right) \delta$ 7.29-7.12 (m, 7H), 6.83-6.81 (m, $2 \mathrm{H}), 4.10(\mathrm{q}, J=7.2 \mathrm{~Hz}, 1 \mathrm{H}), 3.77(\mathrm{~s}, 3 \mathrm{H}), 1.61(\mathrm{~d}, J=7.2 \mathrm{~Hz}, 3 \mathrm{H}) ;{ }^{13} \mathrm{C}\left\{{ }^{1} \mathrm{H}\right\} \mathrm{NMR}(100$ $\left.\mathrm{MHz}, \mathrm{CDCl}_{3}\right) \delta 157.8,146.8,138.6,128.5,128.3,127.5,125.9,113.7,55.2,43.9,22.1$.

1-Chloro-3-(1-phenylethyl)benzene (7f): Purified by silica gel chromatography (1\% ethyl acetate/99\% hexanes); Clear viscous oil (0.11 g, 92\%); IR (DCM) 3434, 2090, $1642 \mathrm{~cm}^{-1}$; 2968,1594, 1493, 1475, 1426, 1082, 791; TLC $\mathrm{R}_{\mathrm{f}}=0.73$ (10\% ethyl acetate/90\% hexanes);

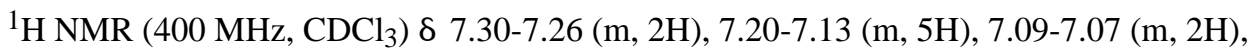
$4.10(\mathrm{q}, J=7.2 \mathrm{~Hz}, 1 \mathrm{H}), 1.61(\mathrm{~d}, J=7.2 \mathrm{~Hz}, 3 \mathrm{H}) ;{ }^{13} \mathrm{C}\left\{{ }^{1} \mathrm{H}\right\} \mathrm{NMR}\left(75 \mathrm{MHz}, \mathrm{CDCl}_{3}\right) \delta 148.5$, 145.5, 134.2, 129.7, 128.6, 127.8, 127.6, 126.4, 126.3, 125.9, 44.6, 21.7. Calcd for $\mathrm{C}_{14} \mathrm{H}_{13} \mathrm{Cl}$ : C, 77.59; H, 6.05; Found: C, 77.33; H, 5.76

1-Bromo-4-(1-phenylethyl)benzene (7g) ${ }^{\mathbf{3 9}}$ : Purified by silica gel chromatography (100\% hexanes); Clear viscous oil $(0.11 \mathrm{~g}, 86 \%)$; $\mathrm{TLC}_{\mathrm{f}}=0.71$ (10\% ethyl acetate/90\% hexanes); ${ }^{1} \mathrm{H}$ NMR $\left(400 \mathrm{MHz}, \mathrm{CDCl}_{3}\right) \delta 7.38(\mathrm{~d}, J=8.4 \mathrm{~Hz}, 2 \mathrm{H}), 7.27(\mathrm{t}, J=7.7 \mathrm{~Hz}, 2 \mathrm{H}), 7.21-7.16$ $(\mathrm{m}, 3 \mathrm{H}), 7.07(\mathrm{~d}, J=8.4 \mathrm{~Hz}, 2 \mathrm{H}), 4.09(\mathrm{q}, J=7.2 \mathrm{~Hz}, 1 \mathrm{H}), 1.60(\mathrm{~d}, J=7.2 \mathrm{~Hz}, 3 \mathrm{H})$; ${ }^{13} \mathrm{C}\left\{{ }^{1} \mathrm{H}\right\} \mathrm{NMR}\left(75 \mathrm{MHz}, \mathrm{CDCl}_{3}\right) \delta 145.7,145.4,131.5,129.4,128.5,127.6,126.3,119.9$, $44.3,21.8$.

1-Fluoro-3-(1-phenylethyl)benzene (7h): Purified by silica gel chromatography (100\% hexanes); Clear viscous oil ( $0.04 \mathrm{~g}, 87 \%)$; $\mathrm{TLC} \mathrm{R}_{\mathrm{f}}=0.66$ (100\% hexanes); ${ }^{1} \mathrm{H}$ NMR (400 $\left.\mathrm{MHz}, \mathrm{CDCl}_{3}\right) \delta$ 7.30-7.26 (m, 2H), 7.23-7.17 (m, 4H), $6.99(\mathrm{~d}, J=7.7 \mathrm{~Hz}, 1 \mathrm{H}), 6.92-6.83$ $(\mathrm{m}, 2 \mathrm{H}), 4.13(\mathrm{q}, J=7.2 \mathrm{~Hz}, 1 \mathrm{H}), 1.62(\mathrm{~d}, J=7.2 \mathrm{~Hz}, 3 \mathrm{H}) ;{ }^{13} \mathrm{C}\left\{{ }^{1} \mathrm{H}\right\} \mathrm{NMR}(75 \mathrm{MHz}$, $\left.\mathrm{CDCl}_{3}\right) \delta 163.0(\mathrm{~d}, J=244.5 \mathrm{~Hz}), 149.0,145.6,129.7,128.5,127.6,126.3,123.3,114.5(\mathrm{~d}$, $J=22.5 \mathrm{~Hz}), 112.8(\mathrm{~d}, J=22.4 \mathrm{~Hz}), 44.5$, 21.7. Anal. Calcd for $\mathrm{C}_{14} \mathrm{H}_{13} \mathrm{~F}: \mathrm{C}, 83.97 ; \mathrm{H}, 6.54$. Found: C, 83.88; H, 6.22. 
1-Nitro-4-(1-phenylethyl)benzene (7i) ${ }^{\mathbf{4 0}}$ : Purified by silica gel chromatography (1\% ethyl acetate/99\% hexanes); Yellow oil ( $0.08 \mathrm{~g}, 66 \%)$; $\mathrm{TLC} \mathrm{R}_{\mathrm{f}}=0.55$ (10\% ethyl acetate/90\% hexanes); ${ }^{1} \mathrm{H}$ NMR $\left(400 \mathrm{MHz}, \mathrm{CDCl}_{3}\right) \delta 8.06(\mathrm{~d}, J=8.8, \mathrm{~Hz}, 2 \mathrm{H}), 7.29(\mathrm{~d}, J=8.6 \mathrm{~Hz}, 2 \mathrm{H})$, $7.24(\mathrm{t}, J=7.1 \mathrm{~Hz}, 2 \mathrm{H}), 7.18-7.12(\mathrm{~m}, 3 \mathrm{H}), 4.18(\mathrm{q}, J=7.2 \mathrm{~Hz}, 1 \mathrm{H}), 1.60(\mathrm{~d}, J=7.2 \mathrm{~Hz}$, $3 \mathrm{H}) ;{ }^{13} \mathrm{C}\left\{{ }^{1} \mathrm{H}\right\} \mathrm{NMR}\left(100 \mathrm{MHz}, \mathrm{CDCl}_{3}\right) \delta 154.1,146.4,144.5,128.7,128.5,127.6,126.7$, 123.7, 44.7, 21.5.

1-(1-Phenylethyl)-3,5-bis(trifluoromethyl)benzene (7j): Purified by silica gel chromatography (100\% hexanes); Clear viscous oil ( $0.13 \mathrm{~g}$, 95\%); TLC $\mathrm{R}_{\mathrm{f}}=0.57$ (100\% hexanes); IR (DCM) 2801, 1420, 1374, 1280, 1173, 1134, 897, $701 \mathrm{~cm}^{-1 ; 1} \mathrm{H}$ NMR (400 $\left.\mathrm{MHz}, \mathrm{CDCl}_{3}\right) \delta 7.71(\mathrm{~s}, 1 \mathrm{H}), 7.76(\mathrm{~s}, 2 \mathrm{H}), 7.32(\mathrm{t}, J=7.2 \mathrm{~Hz}, 2 \mathrm{H}), 7.25-7.18(\mathrm{~m}, 3 \mathrm{H}), 4.26$ $(\mathrm{q}, J=7.2 \mathrm{~Hz}, 1 \mathrm{H}), 1.68(\mathrm{~d}, J=7.2 \mathrm{~Hz}, 3 \mathrm{H}) ;{ }^{13} \mathrm{C}\left\{{ }^{1} \mathrm{H}\right\} \mathrm{NMR}\left(100 \mathrm{MHz}, \mathrm{CDCl}_{3}\right)$ 148.9, $144.2,131.7$ (q, $J=44.6 \mathrm{~Hz})$ ), 128.9, 127.8 (q, $J=3.4 \mathrm{~Hz}), 127.5,126.9,123.5$ (q, $J=361.0$ $\mathrm{Hz}$ ), 120.3 (hept, $J=5.2$ ), 44.7, 21.6. Anal. Calcd for $\mathrm{C}_{16} \mathrm{H}_{12} \mathrm{~F}_{6}$ : C, 60.38; H, 3.80. Found: C, $60.65 ; \mathrm{H}, 3.75$.

2-(1-Phenylethyl)-naphthalene (7k $)^{\mathbf{4 1}}$ : Purified by silica gel chromatography (100\% hexanes); Clear viscous oil ( $0.07 \mathrm{~g}, 57 \%)$; $\mathrm{TLC} \mathrm{R}_{\mathrm{f}}=0.52$ (100\% hexanes); ${ }^{1} \mathrm{H}$ NMR (400 $\left.\mathrm{MHz}, \mathrm{CDCl}_{3}\right) \delta$ 7.79-7.76 (m, 2H), $7.72(\mathrm{~d}, J=8.5 \mathrm{~Hz}, 1 \mathrm{H}), 7.69(\mathrm{~s}, 1 \mathrm{H}), 7.42-7.38(\mathrm{~m}$, $2 \mathrm{H}), 7.30-7.24(\mathrm{~m}, 5 \mathrm{H}), 7.20-7.16(\mathrm{~m}, 1 \mathrm{H}), 4.30(\mathrm{q}, J=7.2 \mathrm{~Hz}, 1 \mathrm{H}), 1.72(\mathrm{~d}, J=7.2 \mathrm{~Hz}$, $3 \mathrm{H}) ;{ }^{13} \mathrm{C}\left\{{ }^{1} \mathrm{H}\right\} \operatorname{NMR}\left(75 \mathrm{MHz}, \mathrm{CDCl}_{3}\right) \delta 146.3,143.8,133.6,132.2,128.5,128.0,127.81$, $127.78,127.62,126.9,126.2,126.0,125.4,44.9,21.8$ (one aromatic resonance was not resolved).

1-(1-Phenethyl)-naphthalene (71) ${ }^{\mathbf{4 2}}$ : Purified by silica gel chromatography (2\% ethyl acetate/98\% hexanes); Clear viscous oil ( $0.07 \mathrm{~g}, 57 \%)$; TLC $\mathrm{R}_{\mathrm{f}}=0.73$ (10\% ethyl acetate/90\% hexanes). ${ }^{1} \mathrm{H}$ NMR $\left(400 \mathrm{MHz}, \mathrm{CDCl}_{3}\right) \delta$ 8.02-7.04 $(\mathrm{m}, 1 \mathrm{H}), 7.84-7.82(\mathrm{~m}, 1 \mathrm{H})$, $7.73(\mathrm{~d}, J=7.6 \mathrm{~Hz}, 1 \mathrm{H}), 7.47-7.39(\mathrm{~m}, 4 \mathrm{H}), 7.25-7.22(\mathrm{~m}, 4 \mathrm{H}), 7.17-7.13(\mathrm{~m}, 1 \mathrm{H}), 4.91(\mathrm{q}, J$ $=7.1 \mathrm{~Hz}, 1 \mathrm{H}), 1.76(\mathrm{~d}, J=7.1 \mathrm{~Hz}, 3 \mathrm{H}) .{ }^{13} \mathrm{C}\left\{{ }^{1} \mathrm{H}\right\} \mathrm{NMR}\left(100 \mathrm{MHz}, \mathrm{CDCl}_{3}\right) \delta 146.6,141.5$, 134.0, 131.7, 128.7, 128.4, 127.6, 127.0, 125.9, 125.8, 125.4, 125.3, 124.3, 123.9, 40.5, 22.5.

3-(1-Phenylethyl)-benzo[b]thiophene (7m) ${ }^{\mathbf{4 3}}$ : Purified by silica gel chromatography (2\% ethyl acetate/98\% hexanes); White foam $(0.07 \mathrm{~g}, 57 \%)$; $\mathrm{TLC} \mathrm{R}_{\mathrm{f}}=0.38$ (100\% hexanes); ${ }^{1} \mathrm{H}$ $\operatorname{NMR}\left(400 \mathrm{MHz}, \mathrm{CDCl}_{3}\right) \delta$ 7.83-7.81 (m, 1H), 7.55-7.53 (m, 1H), 7.29-7.15 (m, 8H), 4.43 $(\mathrm{q}, J=7.1 \mathrm{~Hz}, 1 \mathrm{H}), 1.73(\mathrm{~d}, J=7.1 \mathrm{~Hz}, 3 \mathrm{H}) ;{ }^{13} \mathrm{C}\left\{{ }^{1} \mathrm{H}\right\} \mathrm{NMR}\left(75 \mathrm{MHz}, \mathrm{CDCl}_{3}\right) \delta 145.4$, 140.7, 140.6, 138.6, 128.6, 127.4, 126.3, 124.2, 123.8, 122.8, 122.5, 121.5, 39.6, 22.4.

3-(1-(4-Fluorophenyl)ethyl)-benzo[b]thiophene (7n) ${ }^{\mathbf{1 4}} \mathbf{a}$ : Purified by silica gel chromatography (100\% hexanes); Clear viscous oil (0.09 g, 71\%); TLC $\mathrm{R}_{\mathrm{f}}=0.76(10 \%$ ethyl acetate/90\% hexanes); ${ }^{1} \mathrm{H}$ NMR $\left(400 \mathrm{MHz}, \mathrm{CDCl}_{3}\right) \delta 7.80$ (d, $\left.J=7.5 \mathrm{~Hz}, 1 \mathrm{H}\right), 7.49$ (d, $J=7.7 \mathrm{~Hz}, 1 \mathrm{H}), 7.27-7.13(\mathrm{~m}, 5 \mathrm{H}), 6.91(\mathrm{t}, J=8.7 \mathrm{~Hz}, 2 \mathrm{H}), 4.39,(\mathrm{q}, J=7.0 \mathrm{~Hz}, 1 \mathrm{H})$, $1.67(\mathrm{~d}, J=7.1 \mathrm{~Hz}, 3 \mathrm{H}) ;{ }^{13} \mathrm{C}\left\{{ }^{1} \mathrm{H}\right\} \mathrm{NMR}\left(75 \mathrm{MHz}, \mathrm{CDCl}_{3}\right) \delta 161.5(\mathrm{~d}, J=242.3 \mathrm{~Hz}), 141.2$, 140.8, 140.4, 138.5, 128.9, 124.3, 123.9, 122.9, 122.5, 121.6, 115.4 (d, $J=21.7 \mathrm{~Hz}), 38.9$, 22.5 . 
4-Isopropylbiphenyl (7o) ${ }^{44}$ : Purified by silica gel chromatography (100\% hexanes); Clear viscous oil $(0.10 \mathrm{~g}, 87 \%)$; $\mathrm{TLC} \mathrm{R}_{\mathrm{f}}=0.55\left(100 \%\right.$ hexanes); ${ }^{1} \mathrm{H}$ NMR $\left(400 \mathrm{MHz}, \mathrm{CDCl}_{3}\right) \delta$ $7.57(\mathrm{~d}, J=7.2 \mathrm{~Hz}, 2 \mathrm{H}), 7.51(\mathrm{~d}, J=8.2 \mathrm{~Hz}, 2 \mathrm{H}), 7.40(\mathrm{t}, J=7.4 \mathrm{~Hz}, 2 \mathrm{H}), 7.30(\mathrm{t}, J=7.3$ $\mathrm{Hz}, 3 \mathrm{H}), 2.94$ (hept, $J=6.9 \mathrm{~Hz}, 1 \mathrm{H}), 1.28(\mathrm{~d}, J=7.0 \mathrm{~Hz}, 6 \mathrm{H}) ;{ }^{13} \mathrm{C}\left\{{ }^{1} \mathrm{H}\right\} \mathrm{NMR}(100 \mathrm{MHz}$, $\left.\mathrm{CDCl}_{3}\right) \delta 148.1,141.3,138.8,128.8,127.2,127.1,127.0,126.9,33.9,24.1$.

1-(1-Methylpentyl)-4-phenyl-benzene (7p): Purified by silica gel chromatography (100\% hexanes); Clear viscous oil (0.12 g, 97\%); TLC $\mathrm{R}_{\mathrm{f}}=0.64$ (100\% hexanes); IR (DCM) 3027, 2957, 2926, 2856, 1486, 1455, 1008, 837, $732 \mathrm{~cm}^{-1 ;}{ }^{1} \mathrm{H} \mathrm{NMR}\left(400 \mathrm{MHz}, \mathrm{CDCl}_{3}\right) \delta$ 7.60-7.58 (m, 2H), $7.52(\mathrm{~d}, J=8.2 \mathrm{~Hz}, 2 \mathrm{H}), 7.42(\mathrm{t}, J=7.4, \mathrm{~Hz}, 2 \mathrm{H}), 7.34-7.30(\mathrm{~m}, 1 \mathrm{H})$, 7.26-7.24 (m, 2H), 2.72 (hextet, $J=6.8 \mathrm{~Hz}, 1 \mathrm{H}), 1.65-1.56(\mathrm{~m}, 2 \mathrm{H}), 1.33-1.15(\mathrm{~m}, 7 \mathrm{H}), 0.87$ $(\mathrm{t}, J=6.9 \mathrm{~Hz}, 3 \mathrm{H}) ;{ }^{13} \mathrm{C}\left\{{ }^{1} \mathrm{H}\right\} \mathrm{NMR}\left(100 \mathrm{MHz}, \mathrm{CDCl}_{3}\right) \delta 147.2,141.2,138.7,128.7,127.4$, 127.0, 126.9, 39.6, 38.2, 30.0, 22.8, 22.3, 14.1 (one aromatic resonance was unresolved). Anal. Calcd for $\mathrm{C}_{18} \mathrm{H}_{22}:$ C, 90.70; H, 9.30. Found: C, 90.79; H, 9.37.

4-(3-methylbutan-2-yl)-1,1'-biphenyl (7q): Purified by silica gel chromatography (100\% hexanes); Clear viscous oil $(0.065 \mathrm{~g}, 90 \%)$; TLC $\mathrm{R}_{\mathrm{f}}=0.45$ (100\% hexanes); IR (DCM) 3416, 2960, 2872, 1598, $1486 \mathrm{~cm}^{-1 ;}{ }_{1}^{\mathrm{H}} \mathrm{NMR}\left(400 \mathrm{MHz}, \mathrm{CDCl}_{3}\right) \delta 7.61(\mathrm{~d}, J=7.7 \mathrm{~Hz}$, $2 \mathrm{H}), 7.53(\mathrm{~d}, J=8.2 \mathrm{~Hz}, 2 \mathrm{H}), 7.44(\mathrm{t}, J=7.4 \mathrm{~Hz}, 2 \mathrm{H}), 7.33(\mathrm{t}, J=7.3 \mathrm{~Hz}, 1 \mathrm{H}), 7.25(\mathrm{~d}, J=$ $9.1 \mathrm{~Hz}, 2 \mathrm{H}$ ), 2.50 (quintet, $J=7.1 \mathrm{~Hz}, 1 \mathrm{H}$ ), 1.81 (octet, $J=6.6 \mathrm{~Hz}, 1 \mathrm{H}$ ), 1.29 (d, $J=7.0 \mathrm{~Hz}$, $3 \mathrm{H}), 0.98(\mathrm{~d}, J=6.6 \mathrm{~Hz}, 3 \mathrm{H}), 0.82(\mathrm{~d}, J=6.6 \mathrm{~Hz}, 3 \mathrm{H}) ;{ }^{13} \mathrm{C}\left\{{ }^{1} \mathrm{H}\right\} \mathrm{NMR}\left(100 \mathrm{MHz}, \mathrm{CDCl}_{3}\right) \delta$ 146.3, 141.2, 138.6, 128.7, 128.1, 127.02, 127.00, 126.8, 46.6, 34.5, 21.3, 20.2, 18.9. Anal. Calcd for $\mathrm{C}_{17} \mathrm{H}_{20}$ : C, 91.01; H, 8.99. Found: C, 91.18; H, 8.75.

2-(1-Methylpropyl)-naphthalene (7r) ${ }^{\mathbf{1 4 a}}$ : Purified by silica gel chromatography (100\% hexanes); Clear viscous oil (0.07 g, 60\%); TLC $\mathrm{R}_{\mathrm{f}}=0.71$ (100\% hexanes); ${ }^{1} \mathrm{H}$ NMR (400 $\left.\mathrm{MHz}, \mathrm{CDCl}_{3}\right) \delta 7.80-7.75(\mathrm{~m}, 3 \mathrm{H}), 7.60(\mathrm{~s}, 1 \mathrm{H}), 7.41(\mathrm{qd}, J=6.9,1.4 \mathrm{~Hz}, 2 \mathrm{H}), 7.33$ (dd, $J=$ 8.5, 1.6 Hz, 1H), 2.75 (sextet, $J=7.0 \mathrm{~Hz}, 1 \mathrm{H}), 1.74-1.62(\mathrm{~m}, 2 \mathrm{H}), 1.31$ (d, $J=6.9 \mathrm{~Hz}, 3 \mathrm{H})$, $0.84(\mathrm{t}, J=7.4 \mathrm{~Hz}, 3 \mathrm{H}) ;{ }^{13} \mathrm{C}\left\{{ }^{1} \mathrm{H}\right\} \mathrm{NMR}\left(100 \mathrm{MHz}, \mathrm{CDCl}_{3}\right) \delta 145.1,133.6,132.2,127.8$, 127.6, 127.5, 125.9, 125.7, 125.2, 125.0, 41.8, 31.0, 21.9, 12.3 .

3-(1-Methylpropyl)-benzo [b] thiophene (7s) ${ }^{14 a}$ : Purified by silica gel chromatography (100\% hexanes); Clear viscous oil $(0.07 \mathrm{~g}, 62 \%)$; $\mathrm{TLC} \mathrm{R}_{\mathrm{f}}=0.65$ (100\% hexanes); ${ }^{1} \mathrm{H}$ NMR $\left(400 \mathrm{MHz}, \mathrm{CDCl}_{3}\right) \delta$ 7.86-7.84 (m, 1H), 7.79-7.77 (m, 1H), 7.38-7.30 (m, 2H), 7.06 (s, 1H), 3.09 (sextet, $J=6.8 \mathrm{~Hz}, 1 \mathrm{H}), 1.89-1.79(\mathrm{~m}, 1 \mathrm{H}), 1.71-1.60(\mathrm{~m}, 1 \mathrm{H}), 1.35(\mathrm{~d}, J=6.9 \mathrm{~Hz}, 3 \mathrm{H})$, $0.93(\mathrm{t}, J=7.4 \mathrm{~Hz}, 3 \mathrm{H}) ;{ }^{13} \mathrm{C}\left\{{ }^{1} \mathrm{H}\right\} \mathrm{NMR}\left(75 \mathrm{MHz}, \mathrm{CDCl}_{3}\right) \delta 142.5,140.7,138.9,124.0$, 123.7, 122.9, 121.9, 119.6, 34.7, 29.8, 20.3, 12.0.

4-Ethyl-1,3-dimethoxybenzene (7t) ${ }^{\mathbf{4 5}}$ : Purified by silica gel chromatography (5\% ethyl acetate/95\% hexanes); Clear viscous oil $(0.08 \mathrm{~g}, 92 \%)$; TLC $\mathrm{R}_{\mathrm{f}}=0.66$ (20 ethyl acetate/80\% hexanes). ${ }^{1} \mathrm{H}$ NMR (400 MHz, $\left.\mathrm{CDCl}_{3}\right) \delta 6.80(\mathrm{~d}, J=8.6 \mathrm{~Hz}, 1 \mathrm{H}), 6.74-6.73(\mathrm{~m}, 2 \mathrm{H}), 3.88$ $(\mathrm{s}, 3 \mathrm{H}), 3.85$ (s, 3H), $2.60(\mathrm{q}, J=7.6 \mathrm{~Hz}, 2 \mathrm{H}), 1.23(\mathrm{t}, J=7.6 \mathrm{~Hz}, 3 \mathrm{H}) .{ }^{13} \mathrm{C}\left\{{ }^{1} \mathrm{H}\right\}$ NMR $(100$ $\left.\mathrm{MHz}, \mathrm{CDCl}_{3}\right) \delta 148.8,147.0,137.0,119.5,111.3,111.3,56.0,55.8,28.5,15.8$.

4-Ethylanisole $(\mathbf{7 u})^{\mathbf{4 6}}$ : Purified by silica gel chromatography (4\% ethyl acetate/96\% hexanes); Clear viscous oil (0.05 g, 52\%); TLC $\mathrm{R}_{\mathrm{f}}=0.78$ (10\% ethyl acetate/90\% hexanes); 
${ }^{1} \mathrm{H}$ NMR $\left(400 \mathrm{MHz}, \mathrm{CDCl}_{3}\right) \delta 7.10(\mathrm{~d}, J=8.5 \mathrm{~Hz}, 2 \mathrm{H}), 6.82(\mathrm{~d}, J=8.6 \mathrm{~Hz}, 2 \mathrm{H}), 3.77(\mathrm{~s}$, $3 \mathrm{H}), 2.58(\mathrm{q}, J=7.6 \mathrm{~Hz}, 2 \mathrm{H}), 1.20(\mathrm{t}, J=7.6 \mathrm{~Hz}, 3 \mathrm{H}) ;{ }^{13} \mathrm{C}\left\{{ }^{1} \mathrm{H}\right\} \mathrm{NMR}\left(100 \mathrm{MHz}, \mathrm{CDCl}_{3}\right) \delta$ $157.7,136.4,128.7,113.8,55.3,28.0,15.9$.

4-Ethylbiphenyl (7v) $)^{\mathbf{4 4}}$ : Purified by silica gel chromatography (1\% ethyl acetate/99\% hexanes); Clear viscous oil ( $0.09 \mathrm{~g}, 81 \%)$; TLC $\mathrm{R}_{\mathrm{f}}=0.68$ (100\% hexanes); ${ }^{1} \mathrm{H}$ NMR (400 $\left.\mathrm{MHz}, \mathrm{CDCl}_{3}\right) 7.59-7.57(\mathrm{~m}, 2 \mathrm{H}), 7.52-7.50(\mathrm{~d}, J=7.5 \mathrm{~Hz}, 2 \mathrm{H}), 7.42(\mathrm{t}, J=7.4 \mathrm{~Hz}, 2 \mathrm{H})$, 7.33-7.26 (m, 3H), 2.69 (q, $J=7.6 \mathrm{~Hz}, 2 \mathrm{H}), 1.27$ (t, $J=7.6 \mathrm{~Hz}, 3 \mathrm{H}) .{ }^{13} \mathrm{C}\left\{{ }^{1} \mathrm{H}\right\}$ NMR (100 $\left.\mathrm{MHz}, \mathrm{CDCl}_{3}\right) \delta 143.4,141.2,138.6,128.7,128.3,127.1,127.0,127.0,28.5$, 15.6.

1-Ethylnapthalene (7w) ${ }^{47}$ : Purified by silica gel chromatography (100\% hexanes); Clear viscous oil $(0.062 \mathrm{~g}, 60 \%)$; $\mathrm{TLC} \mathrm{R}_{\mathrm{f}}=0.66(100 \%$ hexanes $) ;{ }^{1} \mathrm{H} \mathrm{NMR}\left(400 \mathrm{MHz}, \mathrm{CDCl}_{3}\right) \delta$ $8.04(\mathrm{~d}, J=8.2 \mathrm{~Hz}, 1 \mathrm{H}), 7.85-7.83(\mathrm{~m}, 1 \mathrm{H}), 7.69(\mathrm{~d}, J=8.1 \mathrm{~Hz}, 1 \mathrm{H}), 7.52-7.44(\mathrm{~m}, 2 \mathrm{H})$, $7.40(\mathrm{t}, J=7.2 \mathrm{~Hz}, 1 \mathrm{H}), 7.33(\mathrm{~d}, J=6.9 \mathrm{~Hz}, 1 \mathrm{H}), 3.12(\mathrm{q}, J=7.5 \mathrm{~Hz}, 2 \mathrm{H}), 1.38(\mathrm{t}, J=7.6$ $\mathrm{Hz}, 3 \mathrm{H}) ;{ }^{13} \mathrm{C}\left\{{ }^{1} \mathrm{H}\right\}$ NMR $\left(75 \mathrm{MHz}, \mathrm{CDCl}_{3}\right) \delta 140.3,133.9,131.8,128.8,126.4,125.7$, $125.4,124.9,123.8,25.9,15.1$ (one aromatic resonance was unresolved).

2-Ethylnapthalene (7x) ${ }^{\mathbf{4 8}}$ : Purified by silica gel chromatography (100\% hexanes); Clear viscous oil $(0.05 \mathrm{~g}, 49 \%)$; $\mathrm{TLC} \mathrm{R}_{\mathrm{f}}=0.62\left(100 \%\right.$ hexanes); ${ }^{1} \mathrm{H} \mathrm{NMR}\left(400 \mathrm{MHz}, \mathrm{CDCl}_{3}\right) \delta$ 7.79-7.74 (m, 3H), 7.60 (s, 1H), 7.41-7.33 (m, 2H), 7.33 (dd, $J=8.4,1.2 \mathrm{~Hz}, 1 \mathrm{H}), 2.80$ (q, $J$ $=7.6 \mathrm{~Hz}, 2 \mathrm{H}), 1.31(\mathrm{t}, J=7.6 \mathrm{~Hz}, 3 \mathrm{H}) ;{ }^{13} \mathrm{C}\left\{{ }^{1} \mathrm{H}\right\} \operatorname{NMR}\left(100 \mathrm{MHz}, \mathrm{CDCl}_{3}\right) \delta 141.7,133.7$, $131.9,127.8,127.6,127.4,127.1,125.8,125.5,125.0,29.0,15.5$.

3-Ethyl-benzo[b]thiophene (7y) ${ }^{\mathbf{4 9}}$ : Purified by silica gel chromatography (100\% hexanes); Clear viscous oil $(0.08 \mathrm{~g}, 62 \%)$; $\mathrm{TLC} \mathrm{R}_{\mathrm{f}}=0.57$ (100\% hexanes); ${ }^{1} \mathrm{H}$ NMR (400 MHz, $\left.\mathrm{CDCl}_{3}\right) \delta$ 7.85-7.83 (m, 1H), 7.74-7.72 (m, 1H), 7.38-7.30 (m, 2H), 7.06 (s, $\left.1 \mathrm{H}\right), 2.85$ (qd, $J$ $=7.5,1.0 \mathrm{~Hz}, 2 \mathrm{H}), 1.36(\mathrm{t}, J=7.5 \mathrm{~Hz}, 3 \mathrm{H}) ;{ }^{13} \mathrm{C}\left\{{ }^{1} \mathrm{H}\right\} \mathrm{NMR}\left(75 \mathrm{MHz}, \mathrm{CDCl}_{3}\right) \delta 140.6$, 139.1, 138.7, 124.2, 123.8, 122.9, 121.7, 120.2, 21.8, 13.4.

1,2-Dimethyl (2S)-8-(benzenesulfonyl)-3a-chloro-1H,2H,3H,3aH,8H,8aH-pyrrolo[2,3-b] indole -1,2-dicarboxylate (10): Purified by silica gel chromatography (20\% ethyl acetate/80\% hexanes); White solid $(0.06 \mathrm{~g}, 80 \%)$; $[a]_{D}{ }^{24}=+38.2(c=1.2, \mathrm{DCM})$; TLC $\mathrm{R}_{\mathrm{f}}=$ 0.33 (50\% ethyl acetate/50\% hexanes); ${ }^{1} \mathrm{H}$ NMR $\left(400 \mathrm{MHz}, \mathrm{CDCl}_{3}\right) \delta 7.78(\mathrm{~d}, J=7.7 \mathrm{~Hz}$, 2H), $7.58(\mathrm{~d}, J=8.1 \mathrm{~Hz}, 1 \mathrm{H}), 7.49(\mathrm{t}, J=7.3 \mathrm{~Hz}, 1 \mathrm{H}), 7.40-7.34(\mathrm{~m}, 3 \mathrm{H}), 7.22(\mathrm{~d}, J=7.2$ $\mathrm{Hz}, 1 \mathrm{H}), 7.14(\mathrm{t}, J=7.5 \mathrm{~Hz}, 1 \mathrm{H}), 6.20(\mathrm{~s}, 1 \mathrm{H}), 4.66(\mathrm{~d}, J=8.7 \mathrm{~Hz}, 1 \mathrm{H}), 3.70(\mathrm{~s}, 3 \mathrm{H}), 3.13$ (s, $3 \mathrm{H}), 3.09-3.12(\mathrm{~m}, 1 \mathrm{H}), 2.95-2.86(\mathrm{~m}, 1 \mathrm{H}) ;{ }^{13} \mathrm{C}\left\{{ }^{1} \mathrm{H}\right\} \mathrm{NMR}\left(100 \mathrm{MHz}, \mathrm{CDCl}_{3}\right) \delta 170.0$, 154.3, 142.2, 139.0, 133.3, 132.1, 131.3, 128.9, 127.3, 125.8, 124.4, 118.4, 86.3, 71.4, 59.3, 53.1, 52.3, 43.0. HRMS (ESI) (m/z): calcd for $\mathrm{C}_{20} \mathrm{H}_{19} \mathrm{ClN}_{2} \mathrm{O}_{6} \mathrm{SNa}\left(\mathrm{M}+\mathrm{Na}^{+}\right)$: 473.0544. Found: 473.0541.

N-Methyl-N-[3-(thiophen-2-yl)butyl]benzenesulfonamide (16): Purified by silica gel chromatography (20\% ethyl acetate/80\% hexanes); Clear oil ( $0.070 \mathrm{~g}, 49 \%)$, TLC $\mathrm{R}_{\mathrm{f}}=0.62$ (50\% ethyl acetate/50\% hexanes); IR (DCM) 2923, 1446, 1340, 1164, 735, $691 \mathrm{~cm}^{-1 ; 1} \mathrm{H}$ NMR $\left(400 \mathrm{MHz}, \mathrm{CDCl}_{3}\right) \delta 7.67(\mathrm{~d}, J=7.2 \mathrm{~Hz}, 2 \mathrm{H}), 7.49(\mathrm{t}, J=7.3 \mathrm{~Hz}, 1 \mathrm{H}), 7.43(\mathrm{t}, J=7.7$ $\mathrm{Hz}, 2 \mathrm{H}), 7.06-7.04(\mathrm{~m}, 1 \mathrm{H}), 6.84$ (t, $J=3.5 \mathrm{~Hz}, 1 \mathrm{H}), 6.75(\mathrm{~d}, J=3.3 \mathrm{~Hz}, 1 \mathrm{H}), 3.07$ (sextet, $J$ $=6.9 \mathrm{~Hz}, 1 \mathrm{H}), 2.93(\mathrm{td}, J=7.4,1.9 \mathrm{~Hz}, 2 \mathrm{H}), 2.63(\mathrm{~s}, 3 \mathrm{H}), 1.84-1.71(\mathrm{~m}, 2 \mathrm{H}), 1.27(\mathrm{~d}, J=6.9$ 
$\mathrm{Hz}, 3 \mathrm{H}) ;{ }^{13} \mathrm{C}\left\{{ }^{1} \mathrm{H}\right\}$ NMR $\left(100 \mathrm{MHz}, \mathrm{CDCl}_{3}\right) \delta 150.6,137.5,132.5,129.0,127.4,126.6$, 123.1, 122.8, 48.4, 37.0, 34.9, 32.6, 23.1. Anal. Calcd for $\mathrm{C}_{15} \mathrm{H}_{19} \mathrm{NO}_{2} \mathrm{~S}_{2}$ : C, 58.22; H, 6.19; $\mathrm{N}, 4.53$. Found: C, 58.10; H, 6.14; N, 4.89; Chiral HPLC analysis: OJ column (hexane/i$\left.\mathrm{PrOH}=91 / 9,0.9 \mathrm{~mL} / \mathrm{min}, 254 \mathrm{~nm}, 25^{\circ} \mathrm{C}\right): \mathrm{t}_{\mathrm{r}}=20.4,22.2 \mathrm{~min}$.

1-(Benzenesulfonyl)-1,2,3,4-tetrahydropyridin-4-ol (11): IBX (1.170 g, $4.17 \mathrm{mmol})$ was dissolved in dry DMSO $(3.3 \mathrm{ml})$ and warmed to $75{ }^{\circ} \mathrm{C}$ for $5 \mathrm{~min}$. The known 1-

(benzenesulfonyl)-4-piperidone ${ }^{50}(0.500 \mathrm{~g}, 2.08 \mathrm{mmol})$ was added and the reaction was allowed to proceed for $14 \mathrm{~h}$ at $75{ }^{\circ} \mathrm{C}$. The reaction mixture was then poured into sat. aq. $\mathrm{NaHCO}_{3}$ solution and partitioned with ethyl acetate. The organic phase was then filtered through Celite with ethyl acetate, and then the filtrate was washed with water and brine. The organic layer was then dried $\left(\mathrm{Na}_{2} \mathrm{SO}_{4}\right)$, filtered, and concentrated in vacuo. The residue was purified by silica gel chromatography ( $40 \%$ ethyl acetate/60\% hexanes) to provide crude 1(benzenesulfonyl)-2,3-dehydro-4-piperidone as an off-white solid $(0.297 \mathrm{~g}, 60 \%)$. A portion of this solid $(0.200 \mathrm{~g}, 0.83 \mathrm{mmol})$ was then dissolved in $2 \mathrm{~mL}$ of $\mathrm{MeOH}$. The reaction was cooled to $0^{\circ} \mathrm{C}$ and $\mathrm{CeCl}_{3} \cdot 7 \mathrm{H}_{2} \mathrm{O}(0.313 \mathrm{~g}, 0.83 \mathrm{mmol})$ was added followed by $\mathrm{NaBH}_{4}(0.032$ $\mathrm{g}, 0.83 \mathrm{mmol})$. Reaction mixture was then allowed to warm to room temperature. After 30 min the reaction was poured into water and extracted with ethyl acetate (3x). The combined organic layers were then dried $\left(\mathrm{Na}_{2} \mathrm{SO}_{4}\right)$, filtered and concentrated. The residue was purified by silica gel chromatography (50\% ethyl acetate/50\% hexanes) to provide alcohol $\mathbf{1 1}$ as white solid $(0.191 \mathrm{~g}, 95 \%)$

11. TLC $R_{\mathrm{f}}=0.20$ (40\% ethyl acetate/60\% hexanes); IR (DCM) $3398,1643,1446,1170$, $935 \mathrm{~cm}^{-1 ;}{ }_{1} \mathrm{H} \mathrm{NMR}\left(300 \mathrm{MHz}, \mathrm{CDCl}_{3}\right) \delta$ 7.80-7.78 (m, $\left.1 \mathrm{H}\right), 7.63-7.50(\mathrm{~m}, 3 \mathrm{H}), 6.81(\mathrm{~d}, J=$ $8.3 \mathrm{~Hz}, 1 \mathrm{H}), 5.13$ (ddd, $J=8.3,4.8,1.1 \mathrm{~Hz}, 1 \mathrm{H}), 4.12$ (brs, $1 \mathrm{H}), 3.73-3.66(\mathrm{~m}, 1 \mathrm{H}), 3.14$ (td, $J=11.9,3.2 \mathrm{~Hz}, 1 \mathrm{H}), 1.86-1.64(\mathrm{~m}, 3 \mathrm{H}) ;{ }^{13} \mathrm{C}\left\{{ }^{1} \mathrm{H}\right\}$ NMR $\left(75 \mathrm{MHz}, \mathrm{CDCl}_{3}\right) 137.9,133.1$, 129.3, 127.7, 127.0, 108.8, 59.9, 39.2, 30.0. Anal. Calcd for $\mathrm{C}_{11} \mathrm{H}_{13} \mathrm{NO}_{3} \mathrm{~S}: \mathrm{C}, 55.21 ; \mathrm{H}$, 5.48; N, 5.85. Found: C, 54.82; H, 5.40; N, 6.25.

1-(Benzenesulfonyl)-4-methyl-1,2,3,4-tetrahydropyridine (12): Alcohol 11 (0.150 g, $0.632 \mathrm{mmol}$ ) was dissolved in $1.1 \mathrm{~mL}$ of dry DCM under argon. The reaction was cooled to $0{ }^{\circ} \mathrm{C}$ and DBU $(20 \mu \mathrm{L}, 0.131 \mathrm{mmol})$ was added followed by trichloroacetonitrile $(70 \mu \mathrm{L}$, $0.698 \mathrm{mmol}$ ). The reaction mixture was then allowed to warm to rt and stirred for $15 \mathrm{~min}$. In a separate flask $\mathrm{AlCl}_{3}(84 \mathrm{mg}, 0.632 \mathrm{mmol})$ was dissolved in DCM $(2 \mathrm{~mL})$ and $\mathrm{AlMe}_{3}(0.94$ $\mathrm{mL}, 2 \mathrm{M}$ in hexanes, $1.92 \mathrm{mmol}$ ) was added. After $5 \mathrm{~min}$ the solution containing the trimethylaluminum was added to the imidate formed in the first solution at $0^{\circ} \mathrm{C}$. After $5 \mathrm{~min}$ the reaction was quenched with $1 \mathrm{M} \mathrm{HCl}$ and the mixture extracted with DCM (3x). The combined organic layers were then dried $\left(\mathrm{Na}_{2} \mathrm{SO}_{4}\right)$, filtered and concentrated. The residue was purified by silica gel chromatography (15\% ethyl acetate/ $85 \%$ hexanes) to provide alkene $\mathbf{1 2}$ as yellow oil (94 $\mathrm{mg}, 63 \%$ ).

12. $\mathrm{TLC} \mathrm{R}_{\mathrm{f}}=0.25$ (20\% ethyl acetate/80\% hexanes); IR (DCM) 3033, 2974, 2931, 1446, 1328, 1279,1213, 1164, 1105, $980 \mathrm{~cm}^{-1 ;}{ }^{\mathrm{H}} \mathrm{NMR}\left(400 \mathrm{MHz}, \mathrm{CDCl}_{3}\right) \delta 7.83(\mathrm{~d}, J=7.2 \mathrm{~Hz}$, $2 \mathrm{H}$ ), 7.55 (t, $J=7.6 \mathrm{~Hz}, 1 \mathrm{H}), 7.47$ (t, $J=7.6 \mathrm{~Hz}, 2 \mathrm{H}), 5.67-5.57$ (m, 2H), 4.44 (brs, $1 \mathrm{H}$ ), $3.85(\mathrm{dd}, J=5.9,1.4 \mathrm{~Hz}, 1 \mathrm{H}), 3.19-3.12(\mathrm{~m}, 1 \mathrm{H}), 1.97-1.79(\mathrm{~m}, 2 \mathrm{H}), 1.26(\mathrm{~d}, J=6.8 \mathrm{~Hz}$, $3 \mathrm{H}) .{ }^{13} \mathrm{C}\left\{{ }^{1} \mathrm{H}\right\} \mathrm{NMR}\left(100 \mathrm{MHz}, \mathrm{CDCl}_{3}\right) \delta 141.5,132.3,129.4,129.0,126.8,124.4,49.6$, 
37.8, 23.7, 20.4. Anal. Calcd for $\mathrm{C}_{12} \mathrm{H}_{15} \mathrm{NO}_{2} \mathrm{~S}: \mathrm{C}, 60.73 ; \mathrm{H}, 6.37$; N, 5.90. Found: C, 60.46; $\mathrm{H}, 6.46 ; \mathrm{N}, 5.86$.

N-[3S-Hydroxy-3-(thiophen-2-yl)propyl]-N-methylbenzenesulfonamide (14): $(S)$-3(Methylamino)-1-(2-thienyl) propan-1-ol ${ }^{51}(0.200 \mathrm{~g}, 1.17 \mathrm{mmol})$ was dissolved in dry DCM $(5 \mathrm{~mL})$ and cooled to $0{ }^{\circ} \mathrm{C}$. Pyridine $(0.113 \mathrm{ml}, 1.46 \mathrm{mmol})$ was then added followed by benzenesulfonyl chloride $(0.246 \mathrm{mg}, 1.39 \mathrm{mmol})$. The reaction mixture was then allowed to warm to $\mathrm{rt}$ and stirred for $14 \mathrm{~h}$. The reaction mixture was then poured into water and extracted with ethyl acetate (3x). This organic extracts were then dried $\left(\mathrm{Na}_{2} \mathrm{SO}_{4}\right)$, filtered, concentrated in vacuo. Purification by silica gel chromatography (40\% ethyl acetate/60\% hexanes) gave sulfonamide S1 as a white solid $(0.33 \mathrm{~g}, 90 \%)$.

14. TLC $\mathrm{R}_{\mathrm{f}}=0.45$ (40\% ethyl acetate/60\% hexanes); $[a]_{D}^{24}=-7.4(c=0.8$, DCM); IR (DCM) 3499, 2925, 1446, 1331, 1160, 1089, 737, $691 \mathrm{~cm}^{-1 ;}{ }^{1} \mathrm{H}$ NMR $\left(300 \mathrm{MHz}, \mathrm{CDCl}_{3}\right) \delta$ 7.74-7.71 (m, 2H), 7.61-7.48 (m, 3H), $7.30(\mathrm{~d}, J=5.0 \mathrm{~Hz}, 1 \mathrm{H}), 6.98(\mathrm{dd}, J=5.0,3.5 \mathrm{~Hz}$, $1 \mathrm{H}), 6.93(\mathrm{dd}, J=3.4,1.0 \mathrm{~Hz}, 1 \mathrm{H}), 4.49(\mathrm{dd}, J=8.1,5.3 \mathrm{~Hz}, 1 \mathrm{H}), 3.24-3.14(\mathrm{~m}, 1 \mathrm{H})$, 2.85-2.73 (m, 1H), $2.62(\mathrm{~s}, 3 \mathrm{H}), 2.14-2.01(\mathrm{~m}, 1 \mathrm{H}), 1.93-1.82(\mathrm{~m}, 1 \mathrm{H}) .{ }^{13} \mathrm{C}\left\{{ }^{1} \mathrm{H}\right\}$ NMR $(75$ $\left.\mathrm{MHz}, \mathrm{CDCl}_{3}\right) \delta$ 144.7, 137.4, 132.5, 129.0, 127.4, 126.7, 126.4, 125.5, 71.8, 47.3, 36.8, 34.9. Anal. Calcd for $\mathrm{C}_{14} \mathrm{H}_{17} \mathrm{NO}_{3} \mathrm{~S}_{2}$ : C, 54.00; H, 5.50; N, 4.50. Found: C, 53.72; H, 5.82; $\mathrm{N}, 4.38$.

\section{Supplementary Material}

Refer to Web version on PubMed Central for supplementary material.

\section{Acknowledgments}

Financial support was provided in part by the National Institute of General Medical Sciences (R15-GM116054). Acknowledgement is also made to the Donors of the American Chemical Society Petroleum Research Fund for a New Directions award in support of this research (54823-ND1). NMR spectra were obtained at Syracuse University using instrumentation acquired with the assistance of the National Science Foundation (CHE-1229345).

\section{References}

1. (a) Iversen T, Bundle DR. J Chem Soc, Chem Commun. 1981:1240.(b) Wessel H-P, Iversen T, Bundle DR. J Chem Soc Pekin. 1985; 1:2247.(c) Ali IAI, El Ashry ESH, Schmidt RR. Eur J Org Chem. 2003:4121.(d) Howard KT, Duffy BC, Linaburg MR, Chisholm JD. Org Biomol Chem. 2016; 14:1623. [PubMed: 26691695] (e) Kurosu M, Li K. Synthesis. 2009:3633.

2. (a) Schmidt RR, Michel J. J Carbohydr Chem. 1985; 4:141.(b) Armstrong A, Brackenridge I, Jackson RFW, Kirk JM. Tetrahedron Lett. 1988; 29:2483.(c) Thierry J, Yue C, Potier P. Tetrahedron Lett. 1998; 39:1557.(d) Shoji M, Uno T, Kakeya H, Onose R, Shiina I, Osada H, Hayashi Y. J Org Chem. 2005; 70:9905. [PubMed: 16292821] (e) Shah JP, Russo CM, Howard KT, Chisholm JD. Tetrahedron Lett. 2014; 55:1740.(f) Adhikari AA, Shah JP, Howard KT, Russo CM, Wallach DR, Linaburg MR, Chisholm JD. Synlett. 2014:283.

3. (a) Schmidt RR, Stumpp M. Liebigs Ann Chem. 1983:1249.(b) Dere RT, Kumar A, Kumar V, Schmidt RR, Zhu X. J Org Chem. 2011; 76:7539. [PubMed: 21800823] (c) Andrews JS, Pinto BM. Carbohydr Res. 1995; 270:51. [PubMed: 7627988] (d) Fridman M, Belakhov V, Lee LV, Liang FS, Wong CH, Baasov T. Angew Chem Int Ed Engl. 2005; 44:447. [PubMed: 15624157] (e) Repetto E, Manzano VE, Uhrig ML, Varela O. J Org Chem. 2012; 77:253. [PubMed: 22092141] (f) Ali IAI, Zhu X, El Ashry ESH, Schmidt RR. ARKIVOC. 2012:35.(g) Duffy BC, Howard KT, Chisholm JD. 
Tetrahedron Lett. 2015; 56:3301.(h) Piemontesi C, Wang Q, Zhu J. Org Biomol Chem. 2013; 11:1533. [PubMed: 23348749]

4. (a) Arnold JS, Mwenda ET, Nguyen HM. Angew Chem, Int Ed. 2014; 53:3688.(b) Arnold JS, Nguyen HM. Synthesis. 2013; 45:2101.(c) Arnold JS, Stone RF, Nguyen HM. Org Lett. 2010; 12:4580. [PubMed: 20843019] (d) Wallach DR, Chisholm JD. J Org Chem. 2016; 81:8035. [PubMed: 27487402] (e) Wallach DR, Stege PC, Shah JP, Chisholm JD. J Org Chem. 2015; 80:1993. [PubMed: 25568933] (f) Cran J, Vidhani D, Krafft M. Synlett. 2014; 25:1550.(g) Grigorjeva L, Jirgensons A. Eur J Org Chem. 2012; 2012:5307.(h) Grigorjeva L, Jirgensons A. Eur J Org Chem. 2011:2421.

5. (a) Schmidt, RR., Jung, K-H. Oligosaccharide synthesis with trichloroacetimidates. In: Hanessian, S., editor. Preparative Carbohydrate Chemistry. CRC Press; 1997. p. 283(b) Zhu, X., Schmidt, RR. Glycoside synthesis from 1-oxygen-substituted glycosyl imidates. In: Demchenko, AV., editor. Handbook of Chemical Glycosylation. Wiley-VCH; 2008. p. 143

6. (a) Li C, Wang J. J Org Chem. 2007; 72:7431. [PubMed: 17715969] (b) Devineau A, Pousse G, Taillier C, Blanchet J, Rouden J, Dalla V. Adv Synth Catal. 2010; 352:2881.(c) Piemontesi C, Wang Q, Zhu J. Org Biomol Chem. 2013; 11:1533. [PubMed: 23348749]

7. (a) Schmidt RR, Hoffmann M. Tetrahedron Lett. 1982; 23:409.(b) Mahling JA, Schmidt RR. Synthesis. 1993; 1993:325.(c) Schmidt RR, Effenberger G. Liebigs Ann Chem. 1987:825.(d) Mahling JA, Jung KH, Schmidt RR. Liebigs Ann. 1995:461.(e) Mahling JA, Schmidt RR. Liebigs Ann. 1995:467.

8. (a) Hoffmann MG, Schmidt RR. Liebigs Ann Chem. 1985:2403.(b) Ali IAI, El Ashry ESH, Schmidt RR. Tetrahedron. 2004; 60:4773.(c) Ideguchi T, Yamada T, Shirahata T, Hirose T, Sugawara A, Kobayashi Y, Omura S, Sunazuka T. J Am Chem Soc. 2013; 135:12568. [PubMed: 23957424] (d) Yamada T, Ideguchi-Matsushita T, Hirose T, Shirahata T, Hokari R, Ishiyama A, Iwatsuki M, Sugawara A, Kobayashi Y, Otoguro K, Omura S, Sunazuka T. Chem Eur J. 2015; 21:11855. [PubMed: 26147398] (e) Adhikari AA, Chisholm JD. Org Lett. 2016; 18:4100. [PubMed: 27486831]

9. Rosowsky A, Chen H, Fu H, Queener SF. Bioorg Med Chem. 2003; 11:59. [PubMed: 12467708]

10. O'Sullivan AC, Schaetzer JH, Luethy C, Mathews CJ, Elliott C, Pitterna T, Pabba J, Jacob O, Buchholz A, Blythe J. Synthesis and Insecticidal Activity of New Benzyl- and IndanylOxazolines, Thiazolines and Alkoxy-Alkyl-Imidazolines. ACS Symp Ser. 2015; 1204:411. (Discovery and Synthesis of Crop Protection Products).

11. (a) Song S, Zhu SF, Yu YB, Zhou QL. Angew Chem, Int Ed. 2013; 52:1556.(b) Wang Z, Ai F, Wang Z, Zhao W, Zhu G, Lin Z, Sun J. J Am Chem Soc. 2015; 137:383. [PubMed: 25482291] (c) Chen J, Chen C, Ji C, Lu Z. Org Lett. 2016; 18:1594. [PubMed: 26974555]

12. (a) Sun YY, Yi J, Lu X, Zhang ZQ, Xiao B, Fu Y. Chem Commun. 2014; 50:11060.(b) Kuriyama M, Shinozawa M, Hamaguchi N, Matsuo S, Onomura O. J Org Chem. 2014; 79:5921. [PubMed: 24856346] (c) Blum J, Gelman D, Baidossi W, Shakh E, Rosenfeld A, Aizenshtat Z, Wassermann BC, Frick M, Heymer B, Schutte S, Wernik S, Schumann H. J Org Chem. 1997; 62:8681.

13. (a) Shacklady-McAtee DM, Roberts KM, Basch CH, Song Y-G, Watson MP. Tetrahedron. 2014; 70:4257. [PubMed: 25364060] (b) Maity P, Shacklady-McAtee DM, Yap GPA, Sirianni ER, Watson MP. J Am Chem Soc. 2013; 135:280. [PubMed: 23268734]

14. (a) Taylor BLH, Swift EC, Waetzig JD, Jarvo ER. J Am Chem Soc. 2011; 133:389. [PubMed: 21155567] (b) Greene MA, Yonova IM, Williams FJ, Jarvo ER. Org Lett. 2012; 14:4293. [PubMed: 22568515] (c) Wisniewska HM, Swift EC, Jarvo ER. J Am Chem Soc. 2013; 135:9083. [PubMed: 23751004] (d) Tollefson EJ, Dawson DD, Osborne CA, Jarvo ER. J Am Chem Soc. 2014; 136:14951. [PubMed: 25308512] (e) Tollefson EJ, Hanna LE, Jarvo ER. Acc Chem Res. 2015; 48:2344. [PubMed: 26197033]

15. Liu M, Zhang J, Zhou H, Yang H, Xia C, Jiang G. RSC Adv. 2016; 6:76780.

16. (a) Tomooka K, Matsuzawa K, Suzuki K, Tsuchihashi G. Tetrahedron Lett. 1987; 28:6339.(b) Deelertpaiboon P, Reutrakul V, Jarussophon S, Tuchinda P, Kuhakarn C, Pohmakotr M. Tetrahedron Lett. 2009; 50:6233.(c) Fotsch CH, Chamberlin AR. J Org Chem. 1991; 56:4141.(d) Crawley GC, Briggs MT. J Org Chem. 1995; 60:4264.(e) Suzuki, K., Nagasawa, T., Saito, S. In eEROS Encyclopedia of Reagents for Organic Synthesis. John Wiley \& Sons, Ltd; 2001. Trimethylaluminum. 
17. (a) Miller DB. J Org Chem. 1966; 31:908.(b) Tanaka H, Shishido Y. Bioorg Med Chem Lett. 2007; 17:6079. [PubMed: 17919904] (c) Hartsel JA, Craft DT, Chen QH, Ma M, Carlier PR. J Org Chem. 2012; 77:3127. [PubMed: 22394317]

18. Honda Y, Morita E, Tsuchihashi G. Chem Lett. 1986:277.

19. Mahoney SJ, Lou T, Bondarenko G, Fillion E. Org Lett. 2012; 14:3474. [PubMed: 22697467]

20. (a) Carde L, Davies DH, Roberts SM. Perkin. 2000; 1:2455.(b) Jansen R, Knopp M, Amberg W, Bernard H, Koser S, Mueller S, Muenster I, Pfeiffer T, Riechers H. Org Process Res Dev. 2001; 5:16.(c) Favaloro FG, Goudreau CA, Mundy BP, Poon T, Slobodzian SV, Jensen BL. Synth Commun. 2001; 31:1847.

21. (a) Yoshida H, Takada A, Mitsunobu O. Tetrahedron Lett. 1998; 39:3007.(b) Nishimura N, Mitsunobu O. Tetrahedron Lett. 2000; 41:2945.

22. Kennedy JP, Desai NV, Sivaram S. J Amer Chem Soc. 1973; 95:6386.

23. Melby E, Kennedy JP. J Org Chem. 1974; 39:2433.

24. Adhikari AA, Suzuki T, Gilbert RT, Linaburg MR, Chisholm JD. J Org Chem. 2017; 82:3982. [PubMed: 28323428]

25. Zhang J, Schmidt RR. Synlett. 2006:1729.

26. Moree WJ, Li B-F, Jovic F, Coon T, Yu J, Gross RS, Tucci F, Marinkovic D, Zamani-Kord S, Malany S, Bradbury MJ, Hernandez LM, O’Brien Z, Wen J, Wang H, Hoare SRJ, Petroski RE, Sacaan A, Madan A, Crowe PD, Beaton G. J Med Chem. 2009; 52:5307. [PubMed: 19663387]

27. Bruncko M, Crich D, Samy R. J Org Chem. 1994; 59:5543.

28. Ruiz-Sanchis P, Savina SA, Albericio F, Alvarez M. Chem Eur J. 2011; 17:1388. [PubMed: 21268138]

29. (a) Cramer F, Pawelzik K, Lichtenthaler FW. Chem Ber. 1958; 91:1555.(b) Schmidt RR, Stumpp M. Liebigs Ann Chem. 1984:680.

30. Bymaster FP, Beedle EE, Findlay J, Gallagher PT, Krushinski JH, Mitchell S, Robertson DW, Thompson DC, Wallace L, Wong DT. Bioorg Med Chem Lett. 2003; 13:4477. [PubMed: 14643350]

31. (a) Namy JL, Abenhaim D. J Organometal Chem. 1972; 43:95.(b) Li DR, Xia WJ, Tu YQ, Zhang FM, Shi L. Chem Commun. 2003:798.

32. Kim CU, Misco PF, Luh BY, Mansuri MM. Tetrahedron Lett. 1994; 35:3017.

33. Zhang Q, Mixdorft JC, Reynders GJ, Nguyen HM. Tetrahedron. 2015; 71:5932.

34. Tokuyama H, Okano K, Fujiwara H, Noji T, Fukuyama T. Chem Asian J. 2011; 6:560. [PubMed: 20936666]

35. Li CK, Li WB, Wang JB. Tetrahedron Lett. 2009; 50:2533.

36. Huang RF, Zhang XH, Pan J, Li JQ, Shen H, Ling XG, Xiong Y. Tetrahedron. 2015; 71:1540.

37. Agasti S, Dey A, Maiti D. Chem Commun. 2016; 52:12191.

38. Lee SY, Villani-Gale A, Eichman CC. Org Lett. 2016; 18:5034. [PubMed: 27668517]

39. Chatterjee I, Qu Z-W, Grimme S, Oestreich M. Angew Chem, Int Ed. 2015; 54:12158.

40. Shang R, Huang Z, Chu L, Fu Y, Liu L. Org Lett. 2011; 13:4240. [PubMed: 21786835]

41. Semba K, Ariyama K, Zheng H, Kameyama R, Sakaki S, Nakao Y. Angew Chem Int Ed Engl. 2016; 55:6275. [PubMed: 27080165]

42. Zhang W, Chen PH, Liu GS. J Am Chem Soc. 2017; 139:7709. [PubMed: 28562032]

43. Cazorla C, Metay E, Lemaire M. Tetrahedron. 2011; 67:8615.

44. Luan YX, Zhang T, Yao WW, Lu K, Kong LY, Lin YT, Ye M. J Am Chem Soc. 2017; 139:1786. [PubMed: 28112504]

45. Alonso F, Riente P, Yus M. Tetrahedron. 2009; 65:10637.

46. Derosa J, Tran VT, Boulous MN, Chen JS, Engle KM. J Am Chem Soc. 2017; 139:10657. [PubMed: 28738150]

47. Liu X, Hsiao CC, Kalvet I, Leiendecker M, Guo L, Schoenebeck F, Rueping M. Angew Chem Int Ed Engl. 2016; 55:6093. [PubMed: 27062726]

48. Murai M, Nishiyama A, Nishinaka N, Morita H, Takai K. Chem Commun. 2017; 53:9281.

49. Guyon C, Baron M, Lemaire M, Popowycz F, Metay E. Tetrahedron. 2014; 70:2088. 
50. Ellis GL, Amewu R, Sabbani S, Stocks PA, Shone A, Stanford D, Gibbons P, Davies J, Vivas L, Charnaud S, Bongard E, Hall C, Rimmer K, Lozanom S, Jesus M, Gargallo D, Ward SA, O’Neill PM. J Med Chem. 2008; 51:2170. [PubMed: 18341274]

51. Suzuki Y, Iwata M, Yazaki R, Kumagai N, Shibasaki M. J Org Chem. 2012; 77:4496. [PubMed: 22494391] 
<smiles>COC(=O)c1cc(C(C)c2c[nH]c3ccccc23)n2ccccc12</smiles>
antitumor agent<smiles>[R]c1cc([C@H](C)c2cc(OC)c(OC)c(OC)c2)ccc1OC</smiles>

$3 \mathrm{R}=\mathrm{H}$, antiviral agent

$4 \mathrm{R}=\mathrm{OH}$, antitumor agent<smiles>Cc1cccc(C(C)c2ncc[nH]2)c1C</smiles>
(pesticide)<smiles>CCCc1sc2ccccc2c1C(C)c1ccc(F)cc1</smiles>

5 anti-insomnia agent

Figure 1.

Biologically Active 1,1'-Diarylethanes 


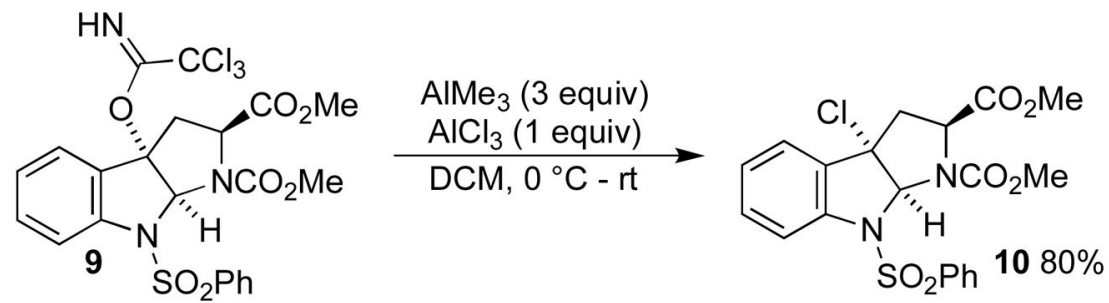

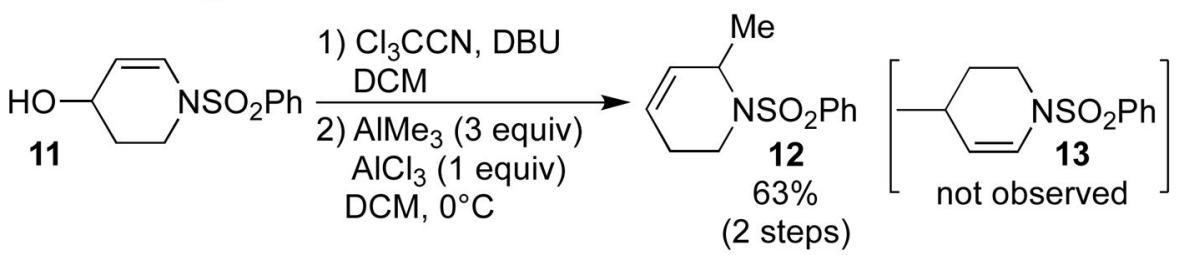

Scheme 1.

Attempted Methylation of 9 and Methylation of 11 


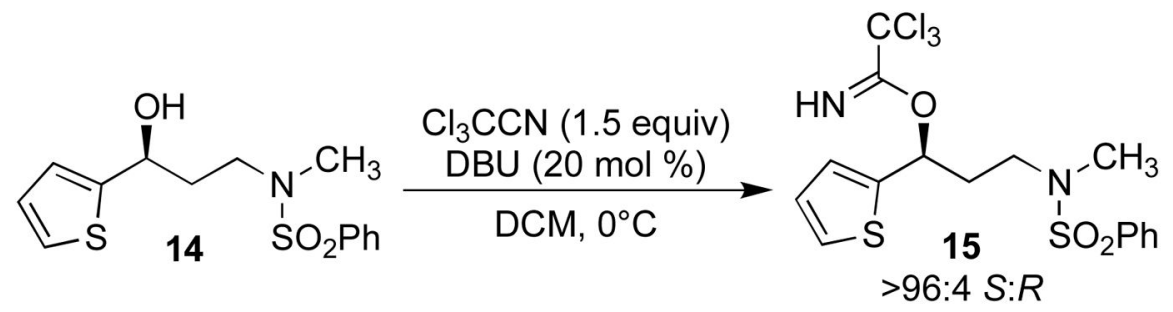

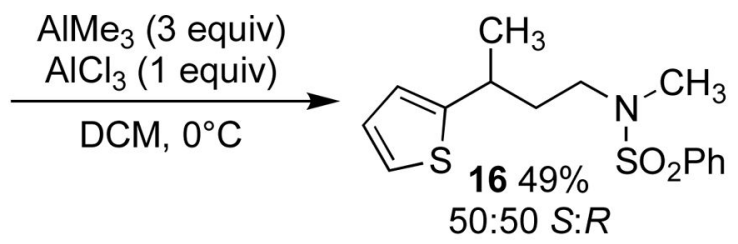

Scheme 2.

Substitution Using a Chiral Trichloroacetimidate 


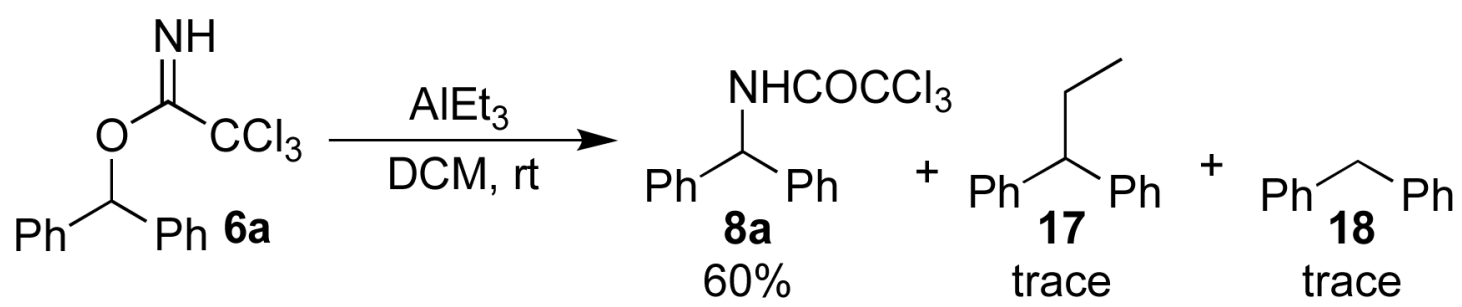

Scheme 3.

Use of Triethylaluminum in the Substitution 
Table 1

Addition of Trimethylaluminum to Trichloroacetimidate $6 \mathrm{a}$

\begin{tabular}{|c|c|c|c|c|}
\hline $\mathrm{Ph}_{\mathrm{Ph}}^{\mathrm{H}}$ & $\frac{\mathrm{AlMe}_{3}}{\text { solvent }}$ & $\left.\sum_{\mathrm{Ph}}^{\mathrm{Ph}}\right\rangle_{7 \mathrm{a}}$ & $\mathrm{Ph}_{\mathrm{Ph}}^{\mathrm{Ph}}$ & $\mathrm{CCl}_{3}$ \\
\hline entry & conditions $^{a}$ & $\operatorname{promoter}^{b}$ & yield $7 \mathbf{a}$ & yield $8 \mathrm{a}$ \\
\hline 1 & toluene, rt, $24 \mathrm{~h}$ & & 0 & 0 \\
\hline 2 & $\mathrm{DCM}, \mathrm{rt}, 4 \mathrm{~h}$ & & 68 & 13 \\
\hline 3 & $\mathrm{Et}_{2} \mathrm{O}, \mathrm{rt}, 24 \mathrm{~h}$ & & 0 & 0 \\
\hline 4 & THF, rt, $18 \mathrm{~h}$ & & 0 & 0 \\
\hline 5 & $\mathrm{MeCN}, \mathrm{rt}, 30 \mathrm{~min}$ & & 0 & 60 \\
\hline 6 & toluene, reflux, $22 \mathrm{~h}$ & & 23 & 13 \\
\hline 7 & DCM, reflux, 22h & & 27 & 25 \\
\hline 8 & THF, reflux, $22 \mathrm{~h}$ & & 0 & 0 \\
\hline $9^{c}$ & $\mathrm{DCM}, \mathrm{rt}, 30 \mathrm{~min}$ & TMSOTf & 0 & 50 \\
\hline $10^{c}$ & $\mathrm{DCM}, \mathrm{rt}, 30 \mathrm{~min}$ & $\mathrm{BF}_{3} \cdot \mathrm{OEt}_{2}$ & $37^{d}$ & $63^{d}$ \\
\hline $11^{c}$ & $\mathrm{DCM}, \mathrm{rt}, 30 \mathrm{~min}$ & $\mathrm{AlCl}_{3}$ & 91 & 8 \\
\hline $12^{c}$ & $\mathrm{DCM}, \mathrm{rt}, 30 \mathrm{~min}$ & $\mathrm{SnCl}_{2}$ & $56^{d}$ & $44^{d}$ \\
\hline $13^{c}$ & $\mathrm{DCM}, \mathrm{rt}, 30 \mathrm{~min}$ & $\mathrm{ZnF}_{2}$ & 68 & 0 \\
\hline $14^{c}$ & $\mathrm{DCM}, \mathrm{rt}, 30 \mathrm{~min}$ & $\mathrm{ZnI}_{2}$ & 68 & 0 \\
\hline $15^{c}$ & $\mathrm{DCM}, \mathrm{rt}, 30 \mathrm{~min}$ & $\mathrm{Sc}(\mathrm{OTf})_{3}$ & 45 & 0 \\
\hline 16 & $\mathrm{DCM}, \mathrm{rt}, 30 \mathrm{~min}$ & $\mathrm{AlCl}_{3}$ & 0 & $60^{e}$ \\
\hline
\end{tabular}

${ }^{a} \mathrm{AlMe} 3$ (1.5 equiv) was added at $0{ }^{\circ} \mathrm{C}$ and then the reaction was warmed to the reported temperature.

${ }^{b}$ One equiv of the Lewis acid was added.

$c_{3}$ Equiv of AlMe3 was used.

$d_{\text {Ratio of products observed in the crude }}{ }^{1} \mathrm{H}$ NMR.

e No AlMe3 was added. 


\section{Table 2}

Addition of Trimethylaluminum to Trichloroacetimidates

\begin{tabular}{|c|c|c|c|}
\hline 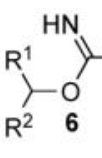 & $\begin{array}{l}\mathrm{AlMe}_{3} \text { (3 equiv) } \\
\mathrm{AlCl}_{3} \text { (1 equiv) } \\
\mathrm{DCM}, 0^{\circ} \mathrm{C}-\mathrm{rt}\end{array}$ & $\sum_{\mathrm{R}^{2}}^{\mathrm{R}^{1}}-\mathrm{Me}$ & $\underset{\mathrm{R}^{2}}{\mathrm{R}_{\mathbf{8}}^{1}} \stackrel{\mathrm{NH}}{\searrow}-\mathrm{CCl}_{3}$ \\
\hline Entry & $\mathbf{R}^{1}$ & $\mathbf{R}^{2}$ & Yield \\
\hline 1 & $\mathrm{Ph}$ & $\mathrm{Ph}$ & $91(\mathbf{7 a})$ \\
\hline 2 & 4-MePh & $\mathrm{Ph}$ & $65(7 b)$ \\
\hline 3 & 2-MePh & $\mathrm{Ph}$ & $70(7 c)$ \\
\hline 4 & 4-(Ph)Ph & $\mathrm{Ph}$ & $91(\mathbf{7 d})$ \\
\hline 5 & 4-OMePh & $\mathrm{Ph}$ & $64^{a}(7 e)$ \\
\hline 6 & 3-ClPh & $\mathrm{Ph}$ & $92(7 f)$ \\
\hline 7 & 4-BrPh & $\mathrm{Ph}$ & $86(7 g)$ \\
\hline 8 & 3-FPh & $\mathrm{Ph}$ & $87(7 h)$ \\
\hline 9 & $4-\mathrm{NO}_{2} \mathrm{Ph}$ & $\mathrm{Ph}$ & $66(7 \mathbf{i})$ \\
\hline 10 & $3,5-\mathrm{CF}_{3} \mathrm{Ph}$ & $\mathrm{Ph}$ & $95(\mathbf{7 j})$ \\
\hline 11 & 2-naphthyl & $\mathrm{Ph}$ & $57(7 \mathbf{k})$ \\
\hline 12 & 1-naphthyl & $\mathrm{Ph}$ & 57 (7l) \\
\hline 13 & 3-benzothienyl & $\mathrm{Ph}$ & $57(7 m)$ \\
\hline 14 & 3-benzothienyl & 4-FPh & $71(7 n)$ \\
\hline 15 & 4-(Ph)Ph & $\mathrm{Me}$ & $87(70)$ \\
\hline 16 & 4-(Ph)Ph & $n-\mathrm{Bu}$ & $97(\mathbf{7 p})$ \\
\hline 17 & 4-(Ph)Ph & $i-\operatorname{Pr}$ & $90^{b}(\mathbf{7 q})$ \\
\hline 18 & 2-naphthyl & Et & $60(7 r)$ \\
\hline 19 & 3-benzothienyl & Et & $62(7 s)$ \\
\hline 20 & 3,4-OMePh & $\mathrm{H}$ & $92^{c, d}(\mathbf{7 t})$ \\
\hline 21 & 4-OMePh & $\mathrm{H}$ & $52^{c, e}(\mathbf{7 u})$ \\
\hline 22 & 4-(Ph)Ph & $\mathrm{H}$ & $81^{c, f}(\mathbf{7 v})$ \\
\hline 23 & 1-naphthyl & $\mathrm{H}$ & $60^{\mathcal{c}, f}(\mathbf{7 w})$ \\
\hline 24 & 2-naphthyl & $\mathrm{H}$ & $49^{c, g}(\mathbf{7} \mathbf{x})$ \\
\hline 25 & 3-benzothienyl & $\mathrm{H}$ & $62^{c, h}(\mathbf{7 y})$ \\
\hline 26 & $4-\mathrm{NO}_{2} \mathrm{Ph}$ & $\mathrm{H}$ & $0(\mathbf{7 z})$ \\
\hline 27 & $\mathrm{Ph}_{2} \mathrm{CH}$ & $\mathrm{H}$ & 0 (7aa) \\
\hline
\end{tabular}

${ }^{a}$ Some rearrangement product $8 \mathbf{e}(28 \%)$ was also isolated.

${ }^{b}$ Reaction started at $-20{ }^{\circ} \mathrm{C}$ and allowed to warm to rt.

$c^{c}$ No aluminium trichloride was added.

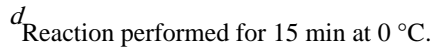


${ }^{e}$ Reaction performed for $15 \min$ at $-20{ }^{\circ} \mathrm{C}$.

$f$ Reaction performed for $4 \mathrm{~h}$ at $0{ }^{\circ} \mathrm{C}$.

$g_{\text {Reaction started at }}-10^{\circ} \mathrm{C}$ and allowed to warm to $5^{\circ} \mathrm{C}$.

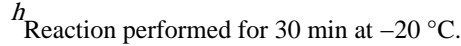

